\title{
FRACTAL DIMENSIONS AND SCALING LAWS IN THE INTERSTELLAR MEDIUM AND GALAXY DISTRIBUTIONS: A NEW FIELD THEORY APPROACH
}

\author{
H. J. de $\operatorname{Vega}^{(a)}$, N. Sánchez ${ }^{(b)}$ and F. Combes ${ }^{(b)}$, \\ (a) Laboratoire de Physique Théorique et Hautes Energies, Université Paris VI, Tour 16, 1er \\ étage, 4, Place Jussieu 75252 Paris, Cedex 05, FRANCE. Laboratoire Associé au CNRS UA 280. \\ (b) Observatoire de Paris, Demirm, 61, Avenue de l'Observatoire, 75014 Paris, FRANCE. \\ Laboratoire Associé au CNRS UA 336, Observatoire de Paris et École Normale Supérieure.
}

(November 1997)

\begin{abstract}
We develop a field theoretical approach to the cold interstellar medium (ISM) and large structure of the universe. We show that a non-relativistic self-gravitating gas in thermal equilibrium with variable number of atoms or fragments is exactly equivalent to a field theory of a single scalar field $\phi(\vec{x})$ with exponential self-interaction. We analyze this field theory perturbatively and non-perturbatively through the renormalization group approach. We show scaling behaviour (critical) for a continuous range of the temperature and of the other physical parameters. We derive in this framework the scaling relation $M(R) \sim R^{d_{H}}$ for the mass on a region of size $R$, and $\Delta v \sim R^{q}$ for the velocity dispersion where $q=\frac{1}{2}\left(d_{H}-1\right)$. For the density-density correlations we find a power-law behaviour for large distances $\sim\left|\vec{r}_{1}-\vec{r}_{2}\right|^{2 d_{H}-6}$. The fractal dimension $d_{H}$ turns to be related with the critical exponent $\nu$ of the correlation length by $d_{H}=1 / \nu$. The renormalization group approach for a single component scalar field in three dimensions states that the long-distance critical behaviour may be governed by the (nonperturbative) Ising fixed point. The Ising values of the scaling exponents are $\nu=0.631 \ldots, d_{H}=1.585 \ldots$ and $q=0.293 \ldots$ Mean field theory yields for the scaling exponents $\nu=1 / 2, d_{H}=2$ and $q=1 / 2$. Both the Ising and the mean field values are compatible with the present ISM observational data: $1.4 \leq d_{H} \leq 2,0.3 \leq q \leq 0.6$. As typical in critical phenomena, the scaling behaviour and critical exponents of the ISM can be obtained without dwelling into the dynamical (time-dependent) behaviour. We develop a field theoretical approach to the galaxy distribution. We consider a gas of selfgravitating masses on the FRW background, in quasi-thermal equilibrium. We show that it exhibits scaling behaviour by renormalization group methods. The galaxy correlations are first computed assuming homogeneity for very large scales and then without assuming homogeneity. In the first case we find $\xi(r) \equiv<\rho\left(\vec{r}_{0}\right) \rho\left(\vec{r}_{0}+\vec{r}\right)>/<\rho>^{2}-1 \sim r^{-\gamma}$, with $\gamma=2$. In the second case we find $D(r)=<\rho\left(\vec{r}_{0}\right) \rho\left(\vec{r}_{0}+\vec{r}\right)>\sim r^{-\Gamma}$ with $\Gamma=1$. While the universe
\end{abstract}


becomes more and more homogeneous at large scales, statistical analysis of galaxy catalogs have revealed a fractal structure at small-scales $\left(\lambda<100 h^{-1}\right.$ Mpc), with a fractal dimension $D=1.5-2$ (Sylos Labini et al 1996).

We study the thermodynamics of a self-gravitating system with the theory of critical phenomena and finite-size scaling and show that gravity provides a dynamical mechanism to produce this fractal structure. Only a limited, (although large), range of scales is involved, between a short-distance cut-off below which other physics intervene, and a large-distance cut-off, where the thermodynamic equilibrium is not satisfied. The galaxy ensemble can be considered at critical conditions, with large density fluctuations developping at any scale. From the theory of critical phenomena, we derive the two independent critical exponents $\nu$ and $\eta$ and predict the fractal dimension $D=1 / \nu$ to be either 1.585 or 2 , depending on whether the long-range behaviour is governed by the Ising or the mean field fixed points, respectively. Both set of values are compatible with present observations. In addition, we predict the scaling behaviour of the gravitational potential to be $r^{-\frac{1}{2}(1+\eta)}$. That is, $r^{-0.5}$ for mean field or $r^{-0.519}$ for the Ising fixed point. The theory allows to compute the three and higher density correlators without any assumption or Ansatz. We find that the connected $N$-points density scales as $r_{1}^{N(D-3)}$, when $r_{1}>>r_{i}, 2 \leq i \leq N$. There are no free parameters in this theory.

\section{THE SELF-INTERACTING GRAVITATIONAL GAS}

We review recent work [1] 3] on the statistical properties of a self-interacting gravitational gas in thermal equilibrium. We discuss two relevant astrophysical applications of the self-interacting gravitational gas: the cold interstellar medium (ISM) and the galaxy distributions.

This review is organized as follows. In section II we summarize the main properties of the ISM and our results for it, in section III we develop the field theory approach to the gravitational gas. A short distance cutoff is naturally present here and prevents zero distance gravitational collapse singularities (which would be unphysical in the present case). Here, the cutoff theory is physically meaningful. The gravitational gas is also treated in a $D$-dimensional space. In section IV we study the scaling behaviour and thermal fluctuations both in perturbation theory and non-perturbatively (renormalization group approach). $g^{2} \equiv$ $\mu T_{\text {eff }}$ acts as the dimensionless coupling constant for the non-linear fluctuations of the field $\phi$. We show that the non-linear fluctuations of the field $\phi$ make the theory to scale ( critical behaviour) for a continuous range of values of the parameters of the theory. Thus, changing the parameters keeps the theory at criticality. The renormalization group analysis made in section IV confirm such results. External forces to the ISM gas like stars are shown not to affect the scaling behaviour of the gas. That is, the scaling exponents $q, d_{H}$ are solely governed by fixed points and hence, they are stable under perturbations.

In section $\mathrm{V}$ we discuss the galaxy distributions starting from the its observational status, in section VI we clarify the definition of the galaxy correlators and then in section VII we develop the dynamical equations in the comoving frame. We apply our field-theory approach 
in section VIII. We derive in this framework two and three point correlators deriving the scaling exponents. Discussion and remarks are presented in section IX.

\section{THE INTERSTELLAR MEDIUM: INTRODUCTION AND RESULTS}

The interstellar medium (ISM) is a gas essentially formed by atomic (HI) and molecular $\left(H_{2}\right)$ hydrogen, distributed in cold $(T \sim 5-50 \mathrm{~K})$ clouds, in a very inhomogeneous and fragmented structure. These clouds are confined in the galactic plane and in particular along the spiral arms. They are distributed in a hierarchy of structures, of observed masses from $1 M_{\odot}$ to $10^{6} M_{\odot}$. The morphology and kinematics of these structures are traced by radio astronomical observations of the $\mathrm{HI}$ hyperfine line at the wavelength of $21 \mathrm{~cm}$, and of the rotational lines of the $\mathrm{CO}$ molecule (the fundamental line being at $2.6 \mathrm{~mm}$ in wavelength), and many other less abundant molecules. Structures have been measured directly in emission from $0.01 \mathrm{pc}$ to $100 \mathrm{pc}$, and there is some evidence in VLBI (very long based interferometry) $\mathrm{HI}$ absorption of structures as low as $10^{-4} p c=20 \mathrm{AU}\left(310^{14} \mathrm{~cm}\right)$. The mean density of structures is roughly inversely proportional to their sizes, and vary between 10 and $10^{5}$ atoms $/ \mathrm{cm}^{3}$ (significantly above the mean density of the ISM which is about 0.1 atoms $/ \mathrm{cm}^{3}$ or $\left.1.610^{-25} \mathrm{~g} / \mathrm{cm}^{3}\right)$. Observations of the ISM revealed remarkable relations between the mass, the radius and velocity dispersion of the various regions, as first noticed by Larson [ [⿴囗十 , and since then confirmed by many other independent observations (see for example ref. [5]). From a compilation of well established samples of data for many different types of molecular clouds of maximum linear dimension (size) $R$, total mass $M$ and internal velocity dispersion $\Delta v$ in each region:

$$
M(R) \sim R^{d_{H}} \quad, \quad \Delta v \sim R^{q},
$$

over a large range of cloud sizes, with $10^{-4}-10^{-2} p c \leq R \leq 100 p c$,

$$
1.4 \leq d_{H} \leq 2,0.3 \leq q \leq 0.6 .
$$

These scaling relations indicate a hierarchical structure for the molecular clouds which is independent of the scale over the above cited range; above $100 \mathrm{pc}$ in size, corresponding to giant molecular clouds, larger structures will be destroyed by galactic shear.

These relations appear to be universal, the exponents $d_{H}, q$ are almost constant over all scales of the Galaxy, and whatever be the observed molecule or element. These properties of interstellar cold gas are supported first at all from observations (and for many different tracers of cloud structures: dark globules using ${ }^{13} \mathrm{CO}$, since the more abundant isotopic species ${ }^{12} \mathrm{CO}$ is highly optically thick, dark cloud cores using $H C N$ or $C S$ as density tracers, giant molecular clouds using ${ }^{12} \mathrm{CO}, \mathrm{HI}$ to trace more diffuse gas, and even cold dust emission in the far-infrared). Nearby molecular clouds are observed to be fragmented and self-similar in projection over a range of scales and densities of at least $10^{4}$, and perhaps up to $10^{6}$.

The physical origin as well as the interpretation of the scaling relations (2.1) are not theoretically understood. The theoretical derivation of these relations has been the subject of many proposals and controversial discussions. It is not our aim here to account for all the proposed models of the ISM and we refer the reader to refs. [5] for a review. 
The physics of the ISM is complex, especially when we consider the violent perturbations brought by star formation. Energy is then poured into the ISM either mechanically through supernovae explosions, stellar winds, bipolar gas flows, etc.. or radiatively through star light, heating or ionising the medium, directly or through heated dust. Relative velocities between the various fragments of the ISM exceed their internal thermal speeds, shock fronts develop and are highly dissipative; radiative cooling is very efficient, so that globally the ISM might be considered isothermal on large-scales. Whatever the diversity of the processes, the universality of the scaling relations suggests a common mechanism underlying the physics.

We propose that self-gravity is the main force at the origin of the structures, that can be perturbed locally by heating sources. Observations are compatible with virialised structures at all scales. Moreover, it has been suggested that the molecular clouds ensemble is in isothermal equilibrium with the cosmic background radiation at $T \sim 3 K$ in the outer parts of galaxies, devoid of any star and heating sources [0]. This colder isothermal medium might represent the ideal frame to understand the role of self-gravity in shaping the hierarchical structures. Our aim is to show that the scaling laws obtained are then quite stable to perturbations.

Till now, no theoretical derivation of the scaling laws eq.(2.1) has been provided in which the values of the exponents are obtained from the theory (and not just taken from outside or as a starting input or hypothesis).

The aim of our work [1] is to develop a theory of the cold ISM. A first step in this goal is to provide a theoretical derivation of the scaling laws eq.(2.1), in which the values of the exponents $d_{H}, q$ are obtained from the theory. For this purpose, we will implement for the ISM the powerful tool of field theory and the Wilson's approach to critical phenomena [15].

We consider a gas of non-relativistic atoms interacting with each other through Newtonian gravity and which are in thermal equilibrium at temperature $T$. We work in the grand canonical ensemble, allowing for a variable number of particles $N$.

Then, we show that this system is exactly equivalent to a field theory of a single scalar field $\phi(\vec{x})$ with exponential interaction. We express the grand canonical partition function $\mathcal{Z}$ as

$$
\mathcal{Z}=\iint \mathcal{D} \phi e^{-S[\phi(.)]},
$$

where

$$
\begin{aligned}
S[\phi(.)] & \equiv \frac{1}{T_{e f f}} \int d^{3} x\left[\frac{1}{2}(\nabla \phi)^{2}-\mu^{2} e^{\phi(\vec{x})}\right], \\
T_{e f f} & =4 \pi \frac{G m^{2}}{T} \quad, \quad \mu^{2}=\sqrt{\frac{2}{\pi}} z G m^{7 / 2} \sqrt{T},
\end{aligned}
$$

$m$ stands for the mass of the atoms and $z$ for the fugacity. We show that in the $\phi$-field language, the particle density expresses as

$$
<\rho(\vec{r})>=-\frac{1}{T_{e f f}}<\nabla^{2} \phi(\vec{r})>=\frac{\mu^{2}}{T_{e f f}}<e^{\phi(\vec{r})}>.
$$

where $\langle\ldots\rangle$ means functional average over $\phi($.$) with statistical weight e^{-S[\phi(.)]}$. Density correlators are written as 


$$
\begin{aligned}
C\left(\vec{r}_{1}, \vec{r}_{2}\right) & \equiv<\rho\left(\vec{r}_{1}\right) \rho\left(\vec{r}_{2}\right)>-<\rho\left(\vec{r}_{1}\right)><\rho\left(\vec{r}_{2}\right)> \\
& =\frac{\mu^{4}}{T_{e f f}^{2}}\left[<e^{\phi\left(\vec{r}_{1}\right)} e^{\phi\left(\vec{r}_{2}\right)}>-<e^{\phi\left(\vec{r}_{1}\right)}><e^{\phi\left(\vec{r}_{2}\right)}>\right]
\end{aligned}
$$

The $\phi$-field defined by eqs.(2.3)-(2.4) has remarkable properties under scale transformations

$$
\vec{x} \rightarrow \vec{x}_{\lambda} \equiv \lambda \vec{x}
$$

where $\lambda$ is an arbitrary real number. For any solution $\phi(\vec{x})$ of the stationary point equations,

$$
\nabla^{2} \phi(\vec{x})+\mu^{2} e^{\phi(\vec{x})}=0
$$

there is a family of dilated solutions of the same equation (2.7), given by

$$
\phi_{\lambda}(\vec{x}) \equiv \phi(\lambda \vec{x})+\log \lambda^{2} .
$$

In addition, $S\left[\phi_{\lambda}().\right]=\lambda^{2-D} S[\phi()$.$] .$

We study the field theory (2.3)-(2.4) both perturbatively and non-perturbatively.

The computation of the thermal fluctuations through the evaluation of the functional integral eq.(2.3) is quite non-trivial. We use the scaling property as a guiding principle. In order to built a perturbation theory in the dimensionless coupling $g \equiv \sqrt{\mu T_{\text {eff }}}$ we look for stationary points of eq.(2.4). We compute the density correlator eq.(2.6) to leading order in $g$. For large distances it behaves as

$$
C\left(\vec{r}_{1}, \vec{r}_{2}\right) \stackrel{\left|\vec{r}_{1}-\vec{r}_{2}\right| \rightarrow \infty}{=} \frac{\mu^{4}}{32 \pi^{2}\left|\vec{r}_{1}-\vec{r}_{2}\right|^{2}}+O\left(\left|\vec{r}_{1}-\vec{r}_{2}\right|^{-3}\right) .
$$

We analyze further this theory with the renormalization group approach. Such nonperturbative approach is the more powerful framework to derive scaling behaviours in field theory 15-17].

We show that the mass contained in a region of size $R$ scales as

$$
<M(R)>=m \int^{R}<e^{\phi(\vec{x})}>d^{3} x \simeq m V a+m \frac{K}{1-\alpha} R^{\frac{1}{\nu}}+\ldots
$$

and the mass fluctuation, $(\Delta M(R))^{2}=<M^{2}>-<M>^{2}$, scales as

$$
\Delta M(R) \sim R^{d_{H}}
$$

Here $\nu$ is the correlation length critical exponent for the $\phi$-theory (2.3) and $a$ and $K$ are constants. Moreover,

$$
<\rho(\vec{r})>=m a+m \frac{K}{4 \pi \nu(1-\alpha)} r^{\frac{1}{\nu}-3} \quad \text { for } r \text { of order } \sim R .
$$

The scaling exponent $\nu$ can be identified with the inverse Haussdorf (fractal) dimension $d_{H}$ of the system

$$
d_{H}=\frac{1}{\nu}
$$


In this way, $M \sim R^{d_{H}}$ according to the usual definition of fractal dimensions [18].

In Fourier space,

$$
<\tilde{\rho}(\vec{k})>\sim k^{-\frac{1}{\nu}} .
$$

From the renormalization group analysis, the density-density correlators (2.6) result to be,

$$
C\left(\vec{r}_{1}, \vec{r}_{2}\right) \sim\left|\vec{r}_{1}-\vec{r}_{2}\right|^{\frac{2}{\nu}-6} .
$$

Computing the average gravitational potential energy and using the virial theorem yields for the velocity dispersion,

$$
\Delta v \sim R^{\frac{1}{2}\left(\frac{1}{\nu}-1\right)} .
$$

This gives a new scaling relation between the exponents $d_{H}$ and $q$

$$
q=\frac{1}{2}\left(\frac{1}{\nu}-1\right)=\frac{1}{2}\left(d_{H}-1\right) .
$$

The perturbative calculation (2.8) yields the mean field value for $\nu$ [13]. That is,

$$
\nu=\frac{1}{2} \quad, \quad d_{H}=2 \quad \text { and } \quad q=\frac{1}{2} .
$$

We find scaling behaviour in the $\phi$-theory for a continuum set of values of $\mu^{2}$ and $T_{\text {eff }}$. The renormalization group transformation amounts to replace the parameters $\mu^{2}$ and $T_{\text {eff }}$ in $\beta H$ and $S[\phi()$.$] by the effective ones at the scale L$ in question.

The renormalization group approach applied to a single component scalar field in three space dimensions indicates that the long distance critical behaviour is governed by the (nonperturbative) Ising fixed point [15 17. Very probably, there are no further fixed points [20]. The scaling exponents associated to the Ising fixed point are

$$
\nu=0.631 \ldots \quad, \quad d_{H}=1.585 \ldots \quad \text { and } \quad q=0.293 \ldots .
$$

Both the mean field (2.12) and the Ising (2.13) numerical values are compatible with the present observational values (2.1) - (2.2).

The theory presented here also predicts a power-law behaviour for the two-points ISM density correlation functionl (see eq.(2.11), $2 d_{H}-6=-2.830 \ldots$, for the Ising fixed point and $2 d_{H}-6=-2$ for the mean field exponents), that should be compared with observations. Previous attempts to derive correlation functions from observations were not entirely conclusive, because of lack of dynamical range [24], but much more extended maps of the ISM could be available soon to test our theory. In addition, we predict an independent exponent for the gravitational potential correlations $\left(\sim r^{-1-\eta}\right.$, where $\eta_{\text {Ising }}=0.037 \ldots$ and $\eta_{\text {mean field }}=0$ [16]), which could be checked through gravitational lenses observations in front of quasars.

The mass parameter $\mu$ [see eq.(2.4)] in the $\phi$-theory turns to coincide at the tree level with the inverse of the Jeans length 


$$
\mu=\sqrt{\frac{12}{\pi}} \frac{1}{d_{J}} .
$$

We find that in the scaling domain the Jeans distance $d_{J}$ grows as $\left\langle d_{J}>\sim R\right.$. This shows that the Jeans distance scales with the size of the system and therefore the instability is present for all sizes $R$. Had $d_{J}$ being of order larger than $R$, the Jeans instability would be absent.

The gravitational gas in thermal equilibrium explains quantitatively the observed scaling laws in the ISM. This fact does not exclude turbulent phenomena in the ISM. Fluid flows (including turbulent regimes) are probably relevant in the dynamics (time dependent processes) of the ISM. As usual in critical phenomena [15, 16], the equilibrium scaling laws can be understood for the ISM without dwelling with the dynamics. A further step in the study of the ISM will be to include the dynamical (time dependent) description within the field theory approach presented in this review.

If the ISM is considered as a flow, the Reynolds number $R e_{I S M}$ on scales $L \sim 100 \mathrm{pc}$ has a very high value of the order of $10^{6}$. This led to the suggestion that the ISM (and the universe in general) could be modelled as a turbulent flow [9]. (Larson [4] first observed that the exponent in the power-law relation for the velocity dispersion is not greatly different from the Kolmogorov value $1 / 3$ for subsonic turbulence).

It must be noticed that the turbulence hypothesis for the ISM is based on the comparison of the ISM with the results known for incompressible flows. However, the physical conditions in the ISM are very different from those of incompressible flows in the laboratory. (And the study of ISM turbulence needs more complete and enlarged investigation than those performed until now based in the concepts of flow turbulence in the laboratory). Besides the facts that the ISM exhibits large density fluctuations on all scales, and the observed fluctuations are highly supersonic, (thus the ISM can not viewed as an 'incompressible' and 'subsonic' flow), and besides other differences, an essential feature to point out is that the long-range self-gravity interaction present in the ISM is completely absent in the studies of flow turbulence. In any case, in a satisfactory theory of the ISM, it should be possible to extract the behaviours of the ISM (be turbulent or whatever) from the theory as a result, instead to be introduced as a starting input or hypothesis.

\section{FIELD THEORY APPROACH TO THE GRAVITATIONAL GAS}

Let us consider a gas of non-relativistic atoms with mass $m$ interacting only through Newtonian gravity and which are in thermal equilibrium at temperature $T \equiv \beta^{-1}$. We shall work in the grand canonical ensemble, allowing for a variable number of particles $N$.

The grand partition function of the system can be written as

$$
\mathcal{Z}=\sum_{N=0}^{\infty} \frac{z^{N}}{N !} \int \ldots \int \prod_{l=1}^{N} \frac{d^{3} p_{l} d^{3} q_{l}}{(2 \pi)^{3}} e^{-\beta H_{N}}
$$

where 


$$
H_{N}=\sum_{l=1}^{N} \frac{p_{l}^{2}}{2 m}-G m^{2} \sum_{1 \leq l<j \leq N} \frac{1}{\left|\overrightarrow{q_{l}}-\vec{q}_{j}\right|}
$$

$G$ is Newton's constant and $z$ is the fugacity.

The integrals over the momenta $p_{l},(1 \leq l \leq N)$ can be performed explicitly in eq.(3.1) using

$$
\int \frac{d^{3} p}{(2 \pi)^{3}} e^{-\frac{\beta p^{2}}{2 m}}=\left(\frac{m}{2 \pi \beta}\right)^{3 / 2}
$$

We thus find,

$$
\mathcal{Z}=\sum_{N=0}^{\infty} \frac{1}{N !}\left[z\left(\frac{m}{2 \pi \beta}\right)^{3 / 2}\right]^{N} \int \ldots \int \prod_{l=1}^{N} d^{3} q_{l} e^{\beta G m^{2} \sum_{1 \leq l<j \leq N} \frac{1}{\left|\vec{q}_{l}-\vec{q}_{j}\right|}}
$$

We proceed now to recast this many-body problem into a field theoretical form |11, 12, 14, 22,

Let us define the density

$$
\rho(\vec{r})=\sum_{j=1}^{N} \delta\left(\vec{r}-\vec{q}_{j}\right)
$$

such that, we can rewrite the potential energy in eq.(3.3) as

$$
\frac{1}{2} \beta G m^{2} \sum_{1 \leq l \neq j \leq N} \frac{1}{\left|\vec{q}_{l}-\vec{q}_{j}\right|}=\frac{1}{2} \beta G m^{2} \int_{|\vec{x}-\vec{y}|>a} \frac{d^{3} x d^{3} y}{|\vec{x}-\vec{y}|} \rho(\vec{x}) \rho(\vec{y}) .
$$

The cutoff $a$ in the r.h.s. is introduced in order to avoid self-interacting divergent terms. However, such divergent terms would contribute to $\mathcal{Z}$ by an infinite multiplicative factor that can be factored out.

By using

$$
\nabla^{2} \frac{1}{|\vec{x}-\vec{y}|}=-4 \pi \delta(\vec{x}-\vec{y})
$$

and partial integration we can now represent the exponent of the potential energy eq.(3.5) as a functional integral [12]

$$
e^{\frac{1}{2} \beta G m^{2} \int \frac{d^{3} x d^{3} y}{|\vec{x}-\vec{y}|} \rho(\vec{x}) \rho(\vec{y})}=\iint \mathcal{D} \xi e^{-\frac{1}{2} \int d^{3} x(\nabla \xi)^{2}+2 m \sqrt{\pi G \beta} \int d^{3} x \xi(\vec{x}) \rho(\vec{x})}
$$

Inserting this expression into eq.(3.3) and using eq.(3.4) yields

$$
\begin{aligned}
& \mathcal{Z}=\sum_{N=0}^{\infty} \frac{1}{N !}\left[z\left(\frac{m}{2 \pi \beta}\right)^{3 / 2}\right]^{N} \iint \mathcal{D} \xi e^{-\frac{1}{2} \int d^{3} x(\nabla \xi)^{2}} \int \ldots \int \prod_{l=1}^{N} d^{3} q_{l} e^{2 m \sqrt{\pi G \beta} \sum_{l=1}^{N} \xi(\vec{q} l)} \\
& =\iint \mathcal{D} \xi e^{-\frac{1}{2} \int d^{3} x(\nabla \xi)^{2}} \sum_{N=0}^{\infty} \frac{1}{N !}\left[z\left(\frac{m}{2 \pi \beta}\right)^{3 / 2}\right]^{N}\left[\int d^{3} q e^{2 m \sqrt{\pi G \beta} \xi(\vec{q})}\right]^{N} \\
& =\iint \mathcal{D} \xi e^{-\int d^{3} x\left[\frac{1}{2}(\nabla \xi)^{2}-z\left(\frac{m}{2 \pi \beta}\right)^{3 / 2} e^{2 m \sqrt{\pi G \beta} \xi(\vec{x})}\right]} .
\end{aligned}
$$


It is convenient to introduce the dimensionless field

$$
\phi(\vec{x}) \equiv 2 m \sqrt{\pi G \beta} \xi(\vec{x}) .
$$

Then,

$$
\mathcal{Z}=\iint \mathcal{D} \phi e^{-\frac{1}{T_{e f f}} \int d^{3} x\left[\frac{1}{2}(\nabla \phi)^{2}-\mu^{2} e^{\phi(\vec{x})}\right]}
$$

where

$$
\mu^{2}=\sqrt{\frac{2}{\pi}} z G m^{7 / 2} \sqrt{T} \quad, \quad T_{e f f}=4 \pi \frac{G m^{2}}{T} .
$$

The partition function for the gas of particles in gravitational interaction has been transformed into the partition function for a single scalar field $\phi(\vec{x})$ with local action

$$
S[\phi(.)] \equiv \frac{1}{T_{\text {eff }}} \int d^{3} x\left[\frac{1}{2}(\nabla \phi)^{2}-\mu^{2} e^{\phi(\vec{x})}\right] .
$$

The $\phi$ field exhibits an exponential self-interaction $-\mu^{2} e^{\phi(\vec{x})}$.

Notice that the effective temperature $T_{\text {eff }}$ for the $\phi$-field partition function turns out to be inversely proportional to $T$ whereas the characteristic length $\mu^{-1}$ behaves as $\sim T^{-1 / 4}$. This is a duality-type mapping between the two models.

It must be noticed that the term $-\mu^{2} e^{\phi(\vec{x})}$ makes the $\phi$-field energy density unbounded from below. Actually, the initial Hamiltonian (3.1) is also unbounded from below. This unboundness physically originates in the attractive character of the gravitational force. Including a short-distance cutoff [see sec. 2A, below] eliminates the zero distance singularity and hence the possibility of zero-distance collapse which is unphysical in the present context. We therefore expect meaningful physical results in the cutoff theory. Moreover, assuming zero boundary conditions for $\phi(\vec{r})$ at $r \rightarrow \infty$ shows that the derivatives of $\phi$ must also be large if $e^{\phi}$ is large. Hence, the term $\frac{1}{2}(\nabla \phi)^{2}$ may stabilize the energy.

The action (3.11) defines a non-renormalizable field theory for any number of dimensions $D>2$. This is a further reason to keep the short-distance cutoff non-zero.

Let us compute now the statistical average value of the density $\rho(\vec{r})$ which in the grand canonical ensemble is given by

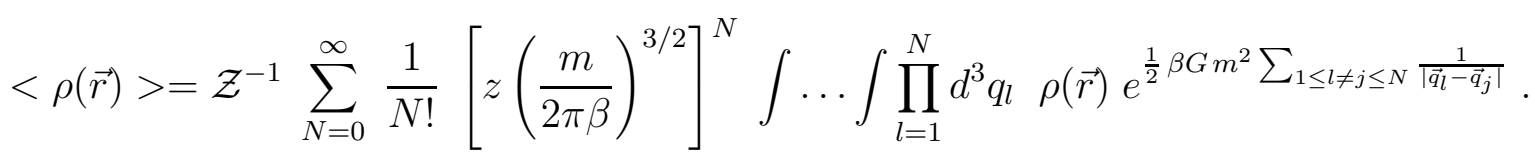

As usual in the functional integral calculations, it is convenient to introduce sources in the partition function (3.9) in order to compute average values of fields

$$
\mathcal{Z}[J(.)] \equiv \iint \mathcal{D} \phi e^{-\frac{1}{T_{e f f}} \int d^{3} x\left[\frac{1}{2}(\nabla \phi)^{2}-\mu^{2} e^{\phi(\vec{x})}\right]+\int d^{3} x J(\vec{x}) \phi(\vec{x})} .
$$

The average value of $\phi(\vec{r})$ then writes as 


$$
<\phi(\vec{r})>=\frac{\delta \log \mathcal{Z}}{\delta J(\vec{r})} .
$$

In order to compute $<\rho(\vec{r})>$ it is useful to introduce

$$
\mathcal{V}[J(.)] \equiv \frac{1}{2} \beta G m^{2} \int_{|\vec{x}-\vec{y}|>a} \frac{d^{3} x d^{3} y}{|\vec{x}-\vec{y}|}[\rho(\vec{x})+J(\vec{x})][\rho(\vec{y})+J(\vec{y})] .
$$

Then, we have

$$
\rho(\vec{r}) e^{\mathcal{V}[0]}=-\left.\frac{1}{T_{e f f}} \nabla_{\vec{x}}^{2}\left(\frac{\delta}{\delta J(\vec{r})} e^{\mathcal{V}[J(.)]}\right)\right|_{J=0} .
$$

By following the same steps as in eqs.(3.6)-(3.7), we find

$$
\begin{aligned}
<\rho(\vec{r})> & =-\frac{1}{T_{\text {eff }}} \nabla_{\vec{r}}^{2}\left(\frac{\delta}{\delta J(\vec{r})} \sum_{N=0}^{\infty} \frac{1}{N !}\left[z\left(\frac{m}{2 \pi \beta}\right)^{3 / 2}\right]^{N} \mathcal{Z}[0]^{-1}\right. \\
\iint \mathcal{D} \xi & \left.e^{-\int d^{3} x\left[\frac{1}{2}(\nabla \xi)^{2}-2 m \sqrt{\pi G \beta} \xi(\vec{x}) J(\vec{x})\right]} \int \ldots \int \prod_{l=1}^{N} d^{3} q_{l} e^{2 m \sqrt{\pi G \beta} \sum_{l=1}^{N} \xi(\vec{q} l)}\right)\left.\right|_{J=0} \\
& =-\left.\frac{1}{T_{e f f}} \nabla_{\vec{r}}^{2}\left(\frac{\delta}{\delta J(\vec{r})} \log \mathcal{Z}[J(.)]\right)\right|_{J=0}
\end{aligned}
$$

Performing the derivatives in the last formula yields

$$
<\rho(\vec{r})>=-\frac{1}{T_{\text {eff }}} \iint \mathcal{D} \phi \nabla^{2} \phi(\vec{r}) e^{-\frac{1}{T_{e f f}} \int d^{3} x\left[\frac{1}{2}(\nabla \phi)^{2}-\mu^{2} e^{\phi(\vec{x})}\right]} \mathcal{Z}[0]^{-1} .
$$

We then see that in the $\phi$-field language the particle density expresses as

$$
\rho(\vec{r})=-\frac{1}{T_{e f f}} \nabla^{2} \phi(\vec{r})
$$

By using eq.(3.18), the gravitational potential at the point $\vec{r}$

$$
U(\vec{r})=-G m \int \frac{d^{3} x}{|\vec{x}-\vec{r}|} \rho(\vec{x})
$$

can be expressed as

$$
U(\vec{r})=-\frac{T}{m} \phi(\vec{r})
$$

We can analogously express the correlation functions as

$$
\begin{aligned}
C\left(\vec{r}_{1}, \vec{r}_{2}\right) & \equiv<\rho\left(\vec{r}_{1}\right) \rho\left(\vec{r}_{2}\right)>-<\rho\left(\vec{r}_{1}\right)><\rho\left(\vec{r}_{2}\right)> \\
& =\left.\left(\frac{1}{T_{e f f}}\right)^{2} \nabla_{\vec{r}_{1}}^{2} \nabla_{\vec{r}_{2}}^{2}\left(\frac{\delta}{\delta J\left(\vec{r}_{1}\right)} \frac{\delta}{\delta J\left(\vec{r}_{2}\right)} \log \mathcal{Z}[J(.)]\right)\right|_{J=0} .
\end{aligned}
$$

This can be also written as 


$$
C\left(\vec{r}_{1}, \vec{r}_{2}\right)=\frac{\mu^{4}}{T_{e f f}^{2}}\left[<e^{\phi\left(\vec{r}_{1}\right)} e^{\phi\left(\vec{r}_{2}\right)}>-<e^{\phi\left(\vec{r}_{1}\right)}><e^{\phi\left(\vec{r}_{2}\right)}>\right] .
$$

A simple short distance regularization of the Newtonian force for the two-body potential is

$$
v_{a}(\vec{r})=-\frac{G m^{2}}{r}[1-\theta(a-r)],
$$

$\theta(x)$ being the step function. The cutoff $a$ can be chosen of the order of atomic distances but its actual value is unessential.

The introduction of the short-distance cutoff elliminates the unphysical short distance collapse of the gravitational gas. please notice, that the interaction between atoms or molecules is repulsive and not gravitational for short distances (Van der Waals forces, for instance).

\section{A. D-dimensional generalization}

This approach generalizes immediately to $D$-dimensional space where the Hamiltonian (3.2) takes then the form

$$
H_{N}=\sum_{l=1}^{N} \frac{p_{l}^{2}}{2 m}-G m^{2} \sum_{1 \leq l<j \leq N} \frac{1}{\left|\vec{q}_{l}-\vec{q}_{j}\right|^{D-2}}, \quad \text { for } D \neq 2
$$

and

$$
H_{N}=\sum_{l=1}^{N} \frac{p_{l}^{2}}{2 m}-G m^{2} \sum_{1 \leq l<j \leq N} \log \frac{1}{\left|\overrightarrow{q_{l}}-\vec{q}_{j}\right|}, \quad \text { at } D=2 \text {. }
$$

The steps from eq.(3.1) to (3.9) can be trivially generalized with the help of the relation

$$
\nabla^{2} \frac{1}{|\vec{x}-\vec{y}|^{D-2}}=-C_{D} \delta(\vec{x}-\vec{y})
$$

in $D$-dimensions and

$$
\nabla^{2} \log \frac{1}{|\vec{x}-\vec{y}|}=-C_{2} \delta(\vec{x}-\vec{y})
$$

at $D=2$.

Here,

$$
C_{D} \equiv(D-2) \frac{2 \pi^{D / 2}}{\Gamma\left(\frac{D}{2}\right)} \text { for } D \neq 2 \text { and } C_{2} \equiv 2 \pi
$$

We finally obtain as a generalization of eq.(3.9),

$$
\mathcal{Z}=\iint \mathcal{D} \phi e^{-\frac{1}{T_{e f f}} \int d^{D} x\left[\frac{1}{2}(\nabla \phi)^{2}-\mu^{2} e^{\phi(\vec{x})}\right]}
$$

where 


$$
\mu^{2}=\frac{C_{D}}{(2 \pi)^{D / 2}} z G m^{2+D / 2} T^{D / 2-1} \quad, \quad T_{e f f}=C_{D} \frac{G m^{2}}{T} .
$$

We have then transformed the partition function for the $D$-dimensional gas of particles in gravitational interaction into the partition function for a scalar field $\phi$ with exponential interaction. The effective temperature $T_{\text {eff }}$ for the $\phi$-field partition function is inversely proportional to $T$ for any space dimension. The characteristic length $\mu^{-1}$ behaves as $\sim$ $T^{-(D-2) / 4}$.

\section{SCALING BEHAVIOUR}

We derive here the scaling behaviour of the $\phi$ field following the general renormalization group arguments in the theory of critical phenomena [15,16]

\section{A. Classical Scale Invariance}

Let us investigate how the action (3.11) transforms under scale transformations

$$
\vec{x} \rightarrow \vec{x}_{\lambda} \equiv \lambda \vec{x}
$$

where $\lambda$ is an arbitrary real number.

In $D$-dimensions the action takes the form

$$
S[\phi(.)] \equiv \frac{1}{T_{\text {eff }}} \int d^{D} x\left[\frac{1}{2}(\nabla \phi)^{2}-\mu^{2} e^{\phi(\vec{x})}\right] .
$$

We define the scale transformed field $\phi_{\lambda}(\vec{x})$ as follows

$$
\phi_{\lambda}(\vec{x}) \equiv \phi(\lambda \vec{x})+\log \lambda^{2}
$$

Hence,

$$
\left(\nabla \phi_{\lambda}(\vec{x})\right)^{2}=\lambda^{2}\left(\nabla_{x_{\lambda}} \phi\left(\vec{x}_{\lambda}\right)\right)^{2} \quad, \quad e^{\phi_{\lambda}(\vec{x})}=\lambda^{2} e^{\phi\left(\vec{x}_{\lambda}\right)}
$$

We find upon changing the integration variable in eq.(4.2) from $\vec{x}$ to $\vec{x}_{\lambda}$

$$
S\left[\phi_{\lambda}(.)\right]=\lambda^{2-D} S[\phi(.)]
$$

We thus see that the action (4.2) scales under dilatations in spite of the fact that it contains the dimensionful parameter $\mu^{2}$. This remarkable scaling property is of course a consequence of the scale behaviour of the gravitational interaction in $D$ dimensions [2].

In particular, in $D=2$ the action (4.2) is scale invariant. In such special case, it is moreover conformal invariant.

The (Noether) current associated to the scale transformations (4.1) is

$$
J_{i}(\vec{x})=x_{j} T_{i j}(\vec{x})+2 \nabla_{i} \phi(\vec{x})
$$


where $T_{i j}(\vec{x})$ is the stress tensor

$$
T_{i j}(\vec{x})=\nabla_{i} \phi(\vec{x}) \nabla_{j} \phi(\vec{x})-\delta_{i j} L
$$

and $L \equiv \frac{1}{2}(\nabla \phi)^{2}-\mu^{2} e^{\phi(\vec{x})}$ stands for the action density. That is,

$$
J_{i}(\vec{x})=(\vec{x} . \nabla \phi+2) \nabla_{i} \phi(\vec{x})-x_{i}\left[\frac{1}{2}(\nabla \phi)^{2}-\mu^{2} e^{\phi(\vec{x})}\right]
$$

By using the classical equation of motion (4.6), we then find

$$
\nabla_{i} J_{i}(\vec{x})=(2-D) L \text {. }
$$

This non-zero divergence is due to the variation of the action under dilatations [eq. (4.4)].

If $\phi(\vec{x})$ is a stationary point of the action (4.2):

$$
\nabla^{2} \phi(\vec{x})+\mu^{2} e^{\phi(\vec{x})}=0
$$

then $\phi_{\lambda}(\vec{x})$ [defined by eq.(4.3)] is also a stationary point:

$$
\nabla^{2} \phi_{\lambda}(\vec{x})+\mu^{2} e^{\phi_{\lambda}(\vec{x})}=0 .
$$

A rotationally invariant stationary point is given by

$$
\phi^{c}(r)=\log \frac{2(D-2)}{\mu^{2} r^{2}} .
$$

This singular solution is invariant under the scale transformations (4.3). That is

$$
\phi_{\lambda}^{c}(r)=\phi^{c}(r)
$$

Eq.(4.7) is dilatation and rotation invariant. It provides the most symmetric stationary point of the action. Notice that there are no constant stationary solutions besides the singular solution $\phi_{0}=-\infty$.

\section{B. Thermal Fluctuations}

In this section we compute the partition function eqs. 3.9) and (3.13) by saddle point methods.

Eq.(4.6) admits only one constant stationary solution

$$
\phi_{0}=-\infty
$$

In order to make such solution finite we now introduce a regularization term $\epsilon \mu^{2} \phi(\vec{x})$ with $\epsilon<<1$ in the action $S$ [eq.(3.11)]. This corresponds to an action density

$$
L=\frac{1}{2}(\nabla \phi)^{2}+u(\phi)
$$


where

$$
u(\phi)=-\mu^{2} e^{\phi(\vec{x})}+\epsilon \mu^{2} \phi(\vec{x}) .
$$

This extra term can be obtained by adding a small constant term $-\epsilon \mu^{2} / T_{\text {eff }}$ to $\rho(\vec{x})$ in eqs.(3.4) - (3.6). This a simple way to make $\phi_{0}$ finite.

We get in this way a constant stationary point at $\phi_{0}=\log \epsilon$ where $u^{\prime}\left(\phi_{0}\right)=0$. However, scale invariance is broken since $u^{\prime \prime}\left(\phi_{0}\right)=-\epsilon \mu^{2} \neq 0$. We can add a second regularization term to $\frac{1}{2} \delta \mu^{2} \phi(\vec{x})^{2}$ to $L$, (with $\delta<<1$ ) in order to enforce $u^{\prime \prime}\left(\phi_{0}\right)=0$. This quadratic term amounts to a long-range shielding of the gravitational force. We finally set:

$$
u(\phi)=-\mu^{2}\left[e^{\phi(\vec{x})}-\epsilon \phi(\vec{x})-\frac{1}{2} \delta \phi(\vec{x})^{2}\right],
$$

where the two regularization parameters $\epsilon$ and $\delta$ are related by

$$
\epsilon(\delta)=\delta[1-\log \delta],
$$

and the stationary point has the value

$$
\phi_{0}=\log \delta
$$

Expanding around $\phi_{0}$

$$
\phi(\vec{x})=\phi_{0}+g \chi(\vec{x})
$$

where $g \equiv \sqrt{\mu^{D-2} T_{e f f}}$ and $\chi(\vec{x})$ is the fluctuation field, yields

$$
\frac{1}{g^{2}} L=\frac{1}{2}(\nabla \chi)^{2}-\frac{\mu^{2} \delta}{g^{2}}\left[e^{g \chi}-1-g \chi-\frac{1}{2} g^{2} \chi^{2}\right]
$$

We see perturbatively in $g$ that $\chi(\vec{x})$ is a massless field.

Concerning the boundary conditions, for the ISM we must consider the system inside a large sphere of radius $R\left(10^{-4}-10^{-2} p c \leq R \leq 100 p c\right)$. That is, all integrals are computed over such large sphere.

Using eq.(3.18) the particle density takes now the form

$$
\rho(\vec{r})=-\frac{1}{T_{e f f}} \nabla^{2} \phi(\vec{r})=-\frac{g}{T_{e f f}} \nabla^{2} \chi(\vec{r})=\frac{\mu^{2} \delta}{T_{e f f}}\left[e^{g \chi(\vec{r})}-1-g \chi(\vec{r})\right] .
$$

It is convenient to renormalize the particle density by its stationary value $\delta=e^{\phi_{0}}$,

$$
\rho(\vec{r})_{r e n} \equiv \frac{1}{\delta} \rho(\vec{r})=\frac{\mu^{D}}{g^{2}}\left[e^{g \chi(\vec{r})}-1-g \chi(\vec{r})\right] .
$$

We see that in the $\delta \rightarrow 0$ limit the interaction in eq.(4.10) vanishes. No infrared divergences appear in the Feynman graphs calculations, since we work on a very large but finite volume of size $R$. Hence, in the $\delta \rightarrow 0$ limit, the whole perturbation series around $\phi_{0}$ reduces to the zeroth order term. 
The constant saddle point $\phi_{0}$ fails to catch up the whole field theory content. In fact, more information arises perturbing around the stationary point $\phi^{c}(r)$ given by eq. (4.7) [23].

Using eqs.(3.21), (4.10) and (4.11) we obtain for the density correlator in the $\delta \rightarrow 0$ limit,

$$
C\left(\vec{r}_{1}, \vec{r}_{2}\right)=\frac{\mu^{2 D}}{g^{4}}\left\{\exp \left[\frac{g^{2}}{C_{D}\left(\mu\left|\vec{r}_{1}-\vec{r}_{2}\right|\right)^{D-2}}\right]-1-\frac{g^{2}}{C_{D}\left(\mu\left|\vec{r}_{1}-\vec{r}_{2}\right|\right)^{D-2}}\right\}
$$

For large distances, we find

$$
C\left(\vec{r}_{1}, \vec{r}_{2}\right) \stackrel{\left|\vec{r}_{1}-\vec{r}_{2}\right|}{=} \rightarrow \infty \frac{\mu^{4}}{2 C_{D}^{2}\left|\vec{r}_{1}-\vec{r}_{2}\right|^{2(D-2)}}+O\left(\left|\vec{r}_{1}-\vec{r}_{2}\right|^{-3(D-2)}\right)
$$

That is, the $\phi$-field theory scales. Namely, the theory behaves critically for a continuum set of values of $\mu$ and $T_{\text {eff }}$.

Notice that the density correlator $C\left(\vec{r}_{1}, \vec{r}_{2}\right)$ behaves for large distances as the correlator of $\chi(\vec{r})^{2}$. This stems from the fact that $\chi(\vec{r})^{2}$ is the most relevant operator in the series expansion of the density (4.11)

$$
\rho(\vec{r})_{r e n}=\frac{1}{2} \mu^{D} \chi(\vec{r})^{2}+O\left(\chi^{3}\right) .
$$

As remarked above, the constant stationary point $\phi_{0}=\log \delta \rightarrow-\infty$ only produces the zeroth order of perturbation theory. More information arises perturbing around the stationary point $\phi^{c}(r)$ given by eq. (4.7) [23].

We succeded in this way to to construct a scale invariant correlations in perturbation theory for the expenential interaction. The existence of such expansion is a necessary condition to show that the theory is scale invariant. Perturbation theory around the stationary point (4.7) shares such scale invariant property [23].

\section{Renormalization Group and Finite Size Scaling Analysis}

As is well known [15 17], physical magnitudes for infinite volume systems diverge at the critical point as $\Lambda$ to a negative power. $\Lambda$ measures the distance to the critical point. (In condensed matter and spin systems, $\Lambda$ is proportional to the temperature minus the critical temperature [16,17]). One has for the correlation length $\xi$,

$$
\xi(\Lambda) \sim \Lambda^{-\nu}
$$

and for the specific heat $\mathcal{C}$,

$$
\mathcal{C} \sim \Lambda^{-\alpha}
$$

Correlation functions scale at criticality. For example, the scalar field $\phi$ (which in spin systems describes the magnetization),

$$
<\phi(\vec{r}) \phi(0)>\sim r^{-1-\eta} .
$$


The critical exponents $\nu, \alpha$ and $\eta$ are pure numbers that depend only on the universality class 15 17.

For a finite volume system, all physical magnitudes are finite at the critical point. Indeed, for a system whose size $R$ is large, the physical magnitudes take large values at the critical point. Thus, for large $R$, one can use the infinite volume theory to treat finite size systems at criticality. In particular, the correlation length provides the relevant physical length $\xi \sim R$. This implies that

$$
\Lambda \sim R^{-1 / \nu} .
$$

We can apply these concepts to the $\phi$-theory since, as we have seen in the previous section, it exhibits scaling in a finite volume $\sim R^{3}$. Namely, the two points correlation function exhibits a power-like behaviour in perturbation theory as shown by eq.(4.12). This happens for a continuum set of values of $T_{\text {eff }}$ and $\mu^{2}$. Therefore, changing $\mu^{2} / T_{\text {eff }}$ keeps the theory in the scaling region. At the point $\mu^{2} / T_{\text {eff }}=0$, the partition function $\mathcal{Z}$ is singular. From eq.(3.10), we shall thus identify

$$
\Lambda \equiv \frac{\mu^{2}}{T_{\text {eff }}}=z\left(\frac{m T}{2 \pi}\right)^{3 / 2} .
$$

Notice that the critical point $\Lambda=0$, corresponds to zero fugacity. [For simplicity, we restrict from now on to the three dimensional case].

Thus, the partition function in the scaling regime can be written as

$$
\mathcal{Z}(\Lambda)=\iint \mathcal{D} \phi e^{-S^{*}+\Lambda} \int d^{3} x e^{\phi(\vec{x})},
$$

where $S^{*}$ stands for the action (3.11) at the critical point $\Lambda=0$.

We define the renormalized mass density as

$$
m \rho(\vec{x})_{r e n} \equiv m e^{\phi(\vec{x})}
$$

and we identify it with the energy density in the renormalization group. [Also called the 'thermal perturbation operator'.] This identification follows from the fact that they are the most relevant positive definite operators. Moreover, such identification is supported by the perturbative result (4.13).

In the scaling regime we have [16] for the logarithm of the partition function

$$
\frac{1}{V} \log \mathcal{Z}(\Lambda)=\frac{K}{(2-\alpha)(1-\alpha)} \Lambda^{2-\alpha}+F(\Lambda),
$$

where $F(\Lambda)$ is an analytic function of $\Lambda$ around the origin

$$
F(\Lambda)=F_{0}+a \Lambda+\frac{1}{2} b \Lambda^{2}+\ldots .
$$

$V=R^{D}$ stands for the volume and $F_{0}, K, a$ and $b$ are constants.

Calculating the logarithmic derivative of $\mathcal{Z}(\Lambda)$ with respect to $\Lambda$ from eqs.4.17) and from (4.19) and equating the results yields 


$$
\frac{1}{V} \frac{\partial}{\partial \Lambda} \log \mathcal{Z}(\Lambda)=a+\frac{K}{1-\alpha} \Lambda^{1-\alpha}+\ldots=\frac{1}{V} \int d^{D} x<e^{\phi(\vec{x})}>.
$$

where we used the scaling relation $\alpha=2-\nu D$ [16, 17].

We can apply here finite size scaling arguments and replace $\Lambda$ by $\sim R^{-\frac{1}{\nu}}$ [eq.44.15)],

$$
\frac{\partial}{\partial \Lambda} \log \mathcal{Z}(\Lambda)=V a+\frac{K}{1-\alpha} R^{1 / \nu}+\ldots .
$$

Recalling eq.(4.18), we can express the mass contained in a region of size $R$ as

$$
M(R)=m \int^{R} e^{\phi(\vec{x})} d^{D} x .
$$

Using eq.(4.20) we find

$$
<M(R)>=m V a+m \frac{K}{1-\alpha} R^{\frac{1}{\nu}}+\ldots .
$$

and

$$
<\rho(\vec{r})>=m a+m \frac{K}{\nu(1-\alpha) \Omega_{D}} r^{\frac{1}{\nu}-D} \quad \text { for } r \text { of order } \sim R .
$$

where $\Omega_{D}$ is the surface of the unit sphere in $D$-dimensions.

The energy density correlators obey the renormalization group equations at criticality [16] - 177. One has for the connected $L$-point correlations of the energy density ('thermal operator'),

$$
\left[\sum_{k=1}^{L} \vec{x}_{k} \cdot \frac{\partial}{\partial \vec{x}_{k}}+L\left(3-\frac{1}{\nu}\right)\right]<\rho\left(\vec{x}_{1}\right) \ldots \rho\left(\vec{x}_{L}\right)>^{c o n n}=0
$$

for the three dimensional space. The homogeneity assumption implies that $\left\langle\rho\left(\vec{x}_{1}\right) \rho\left(\vec{x}_{2}\right)\right\rangle$ is only a function of the difference $\vec{x}_{1}-\vec{x}_{2}$ for large $\left|\vec{x}_{1}-\vec{x}_{2}\right|$. We can therefore write for the density-density correlators (3.20) in 3 space dimensions using by eq.(4.23) for $L=2$,

$$
C\left(\vec{r}_{1}, \vec{r}_{2}\right) \sim\left|\vec{r}_{1}-\vec{r}_{2}\right|^{\frac{2}{\nu}-6} .
$$

where both $\vec{r}_{1}$ and $\vec{r}_{2}$ are inside the finite volume $\sim R^{D}$.

The perturbative calculation (4.12) matches with this result for $\nu=\frac{1}{2}$. That is, the mean field value for the exponent $\nu$.

Let us now compute the second derivative of $\log \mathcal{Z}(\Lambda)$ with respect to $\Lambda$ in two ways. We find from eq.(4.19)

$$
\frac{\partial^{2}}{\partial \Lambda^{2}} \log \mathcal{Z}(\Lambda)=V\left[\Lambda^{-\alpha} K+b+\ldots\right]
$$

We get from eq.(4.17),

$$
\frac{\partial^{2}}{\partial \Lambda^{2}} \log \mathcal{Z}(\Lambda)=\int d^{D} x d^{D} y C(\vec{x}, \vec{y}) \sim R^{D} \int^{R} \frac{d^{3} x}{x^{2 D-2 d_{H}}} \sim \Lambda^{-2} \sim R^{D} \Lambda^{-\alpha}
$$


where we used eq.(4.15), eq.(4.24) and the scaling relation $\alpha=2-\nu D$ [16,17]. We conclude that the scaling behaviours, eq.44.19) for the partition function, eq.(4.14) for the specific heat and eq.(4.24) for the two points correlator are consistent. In addition, eqs.(4.21) and (4.25) yield for the mass fluctuations squared

$$
(\Delta M(R))^{2} \equiv<M^{2}>-<M>^{2} \sim \int d^{D} x d^{D} y C(\vec{x}, \vec{y}) \sim R^{2 d_{H}}
$$

Hence,

$$
\Delta M(R) \sim R^{d_{H}}
$$

The scaling exponent $\nu$ can be identified with the inverse Haussdorf (fractal) dimension $d_{H}$ of the system

$$
d_{H}=\frac{1}{\nu}
$$

In this way, $M \sim R^{d_{H}}$ according to the usual definition of fractal dimensions [18].

The Fourier transform,

$$
\tilde{\rho}(\vec{k}) \equiv \int d^{3} r e^{i \vec{k} \cdot \vec{r}} \rho(\vec{r})
$$

will scale as:

$$
<\tilde{\rho}(\vec{k})>\sim k^{-\frac{1}{\nu}}
$$

This quantity is related to the number of clouds with mass $M, n(M)$, as follows

$$
n(M) \sim<\tilde{\rho}(M)>\sim M^{-x} .
$$

From eqs.(2.10) and (4.28) we find

$$
x=d_{H},
$$

which is in agreement with [6].

Using eq.(4.24) we can compute the average potential energy as

$$
<\mathcal{V}>=\frac{1}{2} \beta G m^{2} \int_{|\vec{x}-\vec{y}|>a}^{R} \frac{d^{3} x d^{3} y}{|\vec{x}-\vec{y}|} C(\vec{x}, \vec{y}) \sim R^{\frac{2}{\nu}-1} .
$$

From here and eq.(4.26) we get as virial estimate for the atoms kinetic energy

$$
<v^{2}>=\frac{<\mathcal{V}>}{M(R)} \sim R^{\frac{1}{\nu}-1}
$$

This corresponds to a velocity dispersion

$$
\Delta v \sim R^{\frac{1}{2}\left(\frac{1}{\nu}-1\right)} .
$$

That is, we predict [see eq.(2.1)] a new scaling relation

$$
q=\frac{1}{2}\left(\frac{1}{\nu}-1\right)=\frac{1}{2}\left(d_{H}-1\right) .
$$


The calculation of the critical amplitudes [that is, the coefficients in front of the powers of $R$ in eqs.(4.26), (4.24) and (4.30) ] is beyond the scope of the present paper [23].

The $\phi$-field connected correlators obey at criticality the renormalization group equation [16] - 17

$$
\left[\sum_{k=1}^{N} \vec{x}_{k} \cdot \frac{\partial}{\partial \vec{x}_{k}}+\frac{N}{2}(1+\eta)\right]<\phi\left(\vec{x}_{1}\right) \ldots \phi\left(\vec{x}_{L}\right)>^{c o n n}=0
$$

for the three dimensional space.

It follows from eq.4.31) that the two-point subtracted correlator of the gravitational potential behaves for long distances as

$$
<\phi\left(\vec{x}_{1}\right) \phi\left(\vec{x}_{2}\right)>\sim\left|\vec{x}_{1}-\vec{x}_{2}\right|^{-1-\eta} .
$$

\section{Values of the scaling exponents and the fractal dimensions}

The scaling exponents $\nu, \alpha$ considered in sec IIIC can be computed through the renormalization group approach. The case of a single component scalar field has been extensively studied in the literature [16,17,20]. Very probably, there is an unique, infrared stable fixed point in three space dimensions: the Ising model fixed point. Such non-perturbative fixed point is reached in the long scale regime independently of the initial shape of the interaction $u(\phi)[$ eq. (4.9)] [20].

The numerical values of the scaling exponents associated to the Ising model fixed point are

$$
\nu=0.631 \ldots \quad, \quad d_{H}=1.585 \ldots \quad \text { and } \quad \alpha=0.107 \ldots
$$

In the $\phi$ field model there are two dimensionful parameters: $\mu$ and $T_{\text {eff }}$. The dimensionless combination

$$
g^{2}=\mu T_{\text {eff }}=(8 \pi)^{3 / 4} \sqrt{z} \frac{G^{3 / 2} m^{15 / 4}}{T^{3 / 4}}
$$

acts as the coupling constant for the non-linear fluctuations of the field $\phi$.

Let us consider a gas formed by neutral hydrogen at thermal equilibrium with the cosmic microwave background. We set $T=2.73 \mathrm{~K}$ and estimate the fugacity $z$ using the ideal gas value

$$
z=\left(\frac{2 \pi}{m T}\right)^{3 / 2} \rho
$$

Here we use $\rho=\delta_{0}$ atoms $\mathrm{cm}^{-3}$ for the ISM density and $\delta_{0} \simeq 10^{10}$. Eq. eqs.(3.10) yields

$$
\frac{1}{\mu}=2.7 \frac{1}{\sqrt{\delta_{0}}} \mathrm{AU} \sim 30 \mathrm{AU} \quad \text { and } \quad g^{2}=\mu T_{e f f}=4.910^{-58} \sqrt{\delta_{0}} \sim 510^{-53}
$$

This extremely low value for $g^{2}$ suggests that the perturbative calculation [sec. IIIB] may apply here yielding the mean field values for the exponents, i. e.

$$
\nu=1 / 2 \quad, \quad d_{H}=2 \quad, \quad \eta=0 \quad \text { and } \quad \alpha=0 .
$$


That is, the effective coupling constant grows with the scale according to the renormalization group flow (towards the Ising fixed point). Now, if the extremely low value of the initial coupling eq. (4.33) applies, the perturbative result (mean field) will hold for many scales (the effective $g$ grows roughly as the length).

$\mu^{-1}$ indicates the order of the smallest distance where the scaling regime applies. A safe lower bound supported by observations is around $20 \mathrm{AU} \sim 3.10^{14} \mathrm{~cm}$, in agreement with our estimate.

Our theoretical predictions for $\Delta M(R)$ and $\Delta v$ [eqs.(4.26) and (4.30)] both for the Ising eq.(4.32) and for the mean field values eq.(4.34), are in agreement with the astronomical observations [eq.(2.1)]. The present observational bounds on the data are larger than the difference between the mean field and Ising values of the exponents $d_{H}$ and $q$.

Further theoretical work in the $\phi$-theory will determine whether the scaling behaviour is given by the mean field or by the Ising fixed point [23].

\section{E. The two dimensional gas and random surfaces fractal dimensions}

In the two dimensional case $(D=2)$ the partition function (3.26) describes the Liouville model that arises in string theory [25] and in the theory of random surfaces (also called twodimensional quantum gravity). For strings in $c$-dimensional Euclidean space the partition function takes the form [25

$$
\mathcal{Z}_{c}=\iint \mathcal{D} \phi e^{-\frac{26-c}{24 \pi} \int d^{2} x\left[\frac{1}{2}(\nabla \phi)^{2}+\mu^{2} e^{\phi(\vec{x})}\right]}
$$

This coincides with eq. 3.26 at $D=2$ provided we flip the sign of $\mu^{2}$ and identify the parameters 3.27 ) as follows,

$$
T=G m^{2} \frac{26-c}{12} \quad, \quad \mu^{2}=z G m^{3}
$$

Ref. [26] states that $d_{H}=4$ for $c \leq 1, d_{H}=3$ for $c=2$ and $d_{H}=2$ for $c \geq 4$. In our context this means

$$
d_{H}=2 \text { for } T \leq \frac{25}{12} G m^{2} \quad, \quad d_{H}=3 \text { for } T=2 G m^{2} \quad \text { and } \quad d_{H}=4 \text { for } T \geq \frac{11}{6} G m^{2} .
$$

For $c \rightarrow \infty, g^{2} \rightarrow 0$ and we can use the perturbative result (4.12) yielding $\nu=\frac{1}{2}, d_{H}=2$ in agreement with the above discussion for $c \geq 4$.

\section{F. Stationary points, the Poisson equation and the Jeans length}

The stationary points of the $\phi$-field partition function (3.9) are given by the non-linear partial differential equation

$$
\nabla^{2} \phi=-\mu^{2} e^{\phi(\vec{x})}
$$

In terms of the gravitational potential $U(\vec{x})$ [see eq. (3.19)], this takes the form 


$$
\nabla^{2} U(\vec{r})=4 \pi G z m\left(\frac{m T}{2 \pi}\right)^{3 / 2} e^{-\frac{m}{T} U(\vec{r})} .
$$

This corresponds to the Poisson equation for a thermal matter distribution fulfilling an ideal gas in hydrostatic equilibrium, as can be seen as follows [19]. The hydrostatic equilibrium condition

$$
\nabla P(\vec{r})=-m \rho(\vec{r}) \nabla U(\vec{r}),
$$

where $P(\vec{r})$ stands for the pressure, combined with the equation of state for the ideal gas

$$
P=T \rho,
$$

yields for the particle density

$$
\rho(\vec{r})=\rho_{0} e^{-\frac{m}{T} U(\vec{r})},
$$

where $\rho_{0}$ is a constant. Inserting this relation into the Poisson equation

$$
\nabla^{2} U(\vec{r})=4 \pi G m \rho(\vec{r})
$$

yields eq. (4.37) with

$$
\rho_{0}=z\left(\frac{m T}{2 \pi}\right)^{3 / 2}
$$

For large $r$, eq.(4.37) gives a density decaying as $r^{-2}$,

$$
\rho(\vec{r}) \stackrel{r \rightarrow \infty}{=} \frac{T}{2 \pi G m} \frac{1}{r^{2}}\left[1+O\left(\frac{1}{\sqrt{r}}\right)\right] \quad, \quad U(\vec{r}) \stackrel{r \rightarrow \infty}{=} \frac{T}{m} \log \left[\frac{2 \pi G \rho_{0}}{T} r^{2}\right]+O\left(\frac{1}{\sqrt{r}}\right) .
$$

Notice that this density, which describes a single stationary solution, decays for large $r$ faster than the density (4.22) governed by thermal fluctuations.

Spherically symmetric solutions of eq.(4.37) has been studied in detail [21]. The small fluctuations around such isothermal spherical solutions as well as the stability problem were studied in [22].

The Jeans distance is in this context,

$$
d_{J} \equiv \sqrt{\frac{3 T}{m}} \frac{1}{\sqrt{G m \rho_{0}}}=\frac{\sqrt{3}(2 \pi)^{3 / 4}}{\sqrt{z G} m^{7 / 4} T^{1 / 4}} .
$$

This distance precisely coincides with $\mu^{-1}$ [see eq.(3.10)] up to an inessential numerical coefficient $(\sqrt{12 / \pi})$. Hence, $\mu$, the only dimensionful parameter in the $\phi$-theory can be interpreted as the inverse of the Jeans distance.

We want to notice that in the critical regime, $d_{J}$ grows as

$$
d_{J} \sim R^{d_{H} / 2}
$$

since $\rho_{0}=\Lambda \sim R^{-d_{H}}$ vanishes as can be seen from eqs.4.15), (4.16) and (4.38). In this estimate we should use for consistency the mean field value $d_{H}=2$, which yields $d_{J} \sim R$.

This shows that the Jeans distance is of the order of the size of the system. The Jeans distance scales and the instability is therefore present for all sizes $R$.

Had $d_{J}$ being of order larger than $R$, the Jeans instability would be absent.

The fact that the Jeans instability is present precisely at $d_{J} \sim R$ is probably essential to the scaling regime and to the self-similar (fractal) structure of the gravitational gas. 


\section{GALAXY DISTRIBUTIONS}

One obvious feature of galaxy and cluster distributions in the sky is their hierarchical property: galaxies gather in groups, that are embedded in clusters, then in superclusters, and so on. (Shapley 1934, Abell 1958). Moreover, galaxies and clusters appear to obey scaling properties, such as the power-law of the two point-correlation function:

$$
\xi(r) \propto r^{-\gamma}
$$

with the slope $\gamma$, the same for galaxies and clusters, of $\approx 1.7$ (e.g. [55]). This scale-invariance has suggested very early the idea of fractal models for the clustering hierachy of galaxies (de Vaucouleurs 1960, 1970; Mandelbrot 1975). Since then, many authors have shown that a fractal distribution indeed reproduces quite well the aspect of galaxy catalogs, for example by simulating a fractal and observing it, as with a telescope (Scott, Shane \& Swanson, 1954; Soneira \& Peebles 1978). Sometimes the analysis has been done in terms of a multifractal medium (Balian \& Schaeffer 1989, Castagnoli \& Provenzale 1991, Martinez et al 1993, Dubrulle \& Lachieze-Rey 1994).

There is some ambiguity in the definition of the two-point correlation function $\xi(r)$ above, since it depends on the assumed scale beyond which the universe is homogeneous; indeed it includes a normalisation by the average density of the universe, which, if the homogeneity scale is not reached, depends on the size of the galaxy sample. Once $\xi(r)$ is defined, one can always determine a length $r_{0}$ where $\xi\left(r_{0}\right)=1$ (Davis \& Peebles 1983, Hamilton 1993). For galaxies, the most frequently reported value is $r_{0} \approx 5 h^{-1} \mathrm{Mpc}$ (where $\left.h=H_{0} / 100 \mathrm{~km} \mathrm{~s}^{-1} \mathrm{Mpc}^{-1}\right)$, but it has been shown to increase with the distance limits of galaxy catalogs (Davis et al 1988). $r_{0}$ is called 'correlation length' in the galaxy literature. [The notion of correlation length $\xi_{0}$ is usually different in physics, where $\xi_{0}$ characterizes the exponential decay of correlations $\left(\sim e^{-r / \xi_{0}}\right)$. For power decaying correlations, it is said that the correlation length is infinite].

The same problem occurs for the two-point correlation function of galaxy clusters; the corresponding $\xi(r)$ has the same power law as galaxies, their length $r_{0}$ has been reported to be about $r_{0} \approx 25 h^{-1} \mathrm{Mpc}$, and their correlation amplitude is therefore about 15 times higher than that of galaxies (Postman, Geller \& Huchra 1986, Postman, Huchra \& Geller 1992). The latter is difficult to understand, unless there is a considerable difference between galaxies belonging to clusters and field galaxies (or morphological segregation). The other obvious explanation is that the normalizing average density of the universe was then chosen lower.

This statistical analysis of the galaxy catalogs has been criticized by Pietronero (1987), Einasto (1989) and Coleman \& Pietronero (1992), who stress the unconfortable dependence of $\xi(r)$ and of the length $r_{0}$ upon the finite size of the catalogs, and on the a priori assumed value of the large-scale homogeneity cut-off. A way to circumvent these problems is to deal instead with the average density as a function of size ( $\mathrm{cf} \S 2$ ). It has been shown that the galaxy distribution behaves as a pure self-similar fractal over scales up to $\approx 100 h^{-1} \mathrm{Mpc}$, the deepest scale to which the data are statistically robust (Sylos Labini et al 1996; Sylos Labini \& Pietronero 1996). This is more consistent with the observation of contrasted large-scale structures, such as superclusters, large voids or great walls of galaxies of $\approx 200 h^{-1} \mathrm{Mpc}$ (de Lapparent et al 1986, Geller \& Huchra 1989). After a proper statistical analysis of all 
available catalogs (CfA, SSRS, IRAS, APM, LEDA, etc.. for galaxies, and Abell and ACO for clusters) Pietronero et al (1997) state that the transition to homogeneity might not yet have been reached up to the deepest scales probed until now. At best, this point is quite controversial, and the large-scale homogeneity transition is not yet well known.

Isotropy and homogeneity are expected at very large scales from the Cosmological Principle (e.g. Peebles 1993). However, this does not imply local or mid-scale homogeneity (e.g. Mandelbrot 1982, Sylos Labini 1994): a fractal structure can be locally isotropic, but inhomogeneous. The main observational evidence in favor of the Cosmological Principle is the remarkable isotropy of the cosmic background radiation (e.g. Smoot et al 1992), that provides information about the Universe at the matter/radiation decoupling. There must therefore exist a transition between the small-scale fractality to large-scale homogeneity. This transition is certainly smooth, and might correspond to the transition from linear perturbations to the non-linear gravitational collapse of structures. The present catalogs do not yet see the transition since they do not look up sufficiently back in time. It can be noticed that some recent surveys begin to see a different power-law behavior at large scales $\left(\lambda \approx 200-400 h^{-1} \mathrm{Mpc}\right.$, e.g. Lin et al 1996).

There are several approaches to understand non-linear clustering, and therefore the distribution of galaxies, in an infinite gravitating system. Numerical simulations have been widely used, in the hope to trace back from the observations the initial mass spectrum of fluctuations, and to test postulated cosmologies such as CDM and related variants (cf Ostriker 1993). This approach has not yet yielded definite results, especially since the physics of the multiple-phase universe is not well known. Also numerical limitations (restricted dynamical range due to the softening and limited volume) have often masked the expected self-similar behavior (Colombi et al 1996). A second approach, which should work essentially in the linear (or weakly non-linear) regime, is to solve the BBGKY hierarchy through closure assumptions (Davis \& Peebles 1977; Balian \& Schaeffer 1989). The main assumption is that the $\mathrm{N}$-points correlation functions are scale-invariant and behave as power-laws like is observed for the few-body correlation functions. Crucial to this approach is the determination of the void probability, which is a series expansion of the $\mathrm{N}$-points correlation functions (White 1979). The hierachical solutions found in this frame agree well with the simulations, and with the fractal structure of the universe at small-scales (Balian \& Schaeffer 1988). A third approach is the thermodynamics of gravitating systems, developped by Saslaw \& Hamilton (1984), which assumes quasi thermodynamic equilibrium. The latter is justified at the small-scales of non-linear clustering, since the expansion time-scale is slow with respect to local relaxation times. Indeed the main effect of expansion is to subtract the mean gravitational field, which is negligible for structures of mean densities several orders of magnitude above average. The predictions of the thermodynamical theory have been successfully compared with N-body simulations (Itoh et al 1993), but a special physical parameter (the ratio of gravitational correlation energy to thermal energy) had to be adjusted for a better fit (Bouchet et al 1991, Sheth \& Saslaw 1996, Saslaw \& Fang 1996).

We present in [3] a new approach based on field theory and the renormalisation group to understand the clustering behaviour of a self-gravitating expanding universe. We also consider the thermodynamics properties of the system, assuming quasi-equilibrium for the range of scales concerned with the non-linear regime and virialisation. We find an exact mapping between the self-gravitating gas and a continuous field theory for a single scalar 
field with an exponential self-coupling. This allows us to use statistical field theory and the renormalisation group to determine the scaling behaviour. The small-scale fractal universe can be considered critical with large density fluctuations developing at any scale. We derive the corresponding critical exponents. They are very close to those measured on galaxy catalogs through statistical methods based on the average density as function of size; these methods reveal in particular a fractal dimension $D \approx 1.5-2$ (Di Nella et al 1996, Sylos Labini \& Amendola 1996, Sylos Labini et al 1996). This fractal dimension is strikingly close to that observed for the interstellar medium or ISM (e.g. Larson 1981, Falgarone et al 1991). We show in ref. [3] that the theoretical framework based on self-gravity that we have developped for the ISM (de Vega, Sánchez \& Combes 1996a,b, hereafter dVSC) is also a dynamical mechanism leading to the small scale fractal structure of the universe. This theory is powerfully predictive without any free parameter. It allows to compute the $N$-points density correlations without any extra assumption.

\section{GALAXY CORRELATION FUNCTIONS AND MASS DENSITY IN A FRACTAL}

The use of the two point correlation function $\xi(r)$ widely spread in galaxy distributions studies, is based on the assumption that the Universe reaches homogeneity on a scale smaller than the sample size. It has been shown by Coleman, Pietronero \& Sanders (1988) and Coleman \& Pietronero (1992) that such an hypothesis could perturb significantly the results. The correlation function is defined as

$$
\xi(r)=\frac{<n\left(r_{i}\right) \cdot n\left(r_{i}+r\right)>}{<n>^{2}}-1
$$

where $n(r)$ is the number density of galaxies, and $\langle\ldots\rangle$ is the volume average (over $d^{3} r_{i}$ ). The length $r_{0}$ is defined by $\xi\left(r_{0}\right)=1$. The function $\xi(r)$ has a power-law behaviour of slope $-\gamma$ for $r<r_{0}$, then it turns down to zero rather quickly at the statitistical limit of the sample. This rapid fall leads to an over-estimate of the small-scale $\gamma$. Pietronero (1987) introduces the conditional density

$$
\Gamma(r)=\frac{<n\left(r_{i}\right) \cdot n\left(r_{i}+r\right)>}{<n>}
$$

which is the average density around an occupied point. For a fractal medium, where the mass depends on the size as

$$
M(r) \propto r^{D}
$$

$D$ being the fractal (Haussdorf) dimension, the conditional density behaves as

$$
\Gamma(r) \propto r^{D-3}
$$

This is exactly the statistical analysis used for the interstellar clouds, since the ISM astronomers have not adopted from the start any large-scale homogeneity assumption (cf Pfenniger \& Combes 1994). 
The fact that for a fractal the correlation $\xi(r)$ can be highly misleading is readily seen since

$$
\xi(r)=\frac{\Gamma(r)}{<n>}-1
$$

and for a fractal structure the average density of the sample $\langle n\rangle$ is a decreasing function of the sample length scale. In the general use of $\xi(r),\langle n\rangle$ is taken for a constant, and we can see that

$$
D=3-\gamma
$$

If for very small scales, both $\xi(r)$ and $\Gamma(r)$ have the same power-law behaviour, with the same slope $-\gamma$, then the slope appears to steepen for $\xi(r)$ when approaching the length $r_{0}$. This explains why with a correct statistical analysis (Di Nella et al 1996, Sylos Labini \& Amendola 1996, Sylos Labini et al 1996), the actual $\gamma \approx 1-1.5$ is smaller than that obtained using $\xi(r)$. This also explains why the amplitude of $\xi(r)$ and $r_{0}$ increases with the sample size, and for clusters as well.

In the following, we adopt the framework of the fractal medium that we used for the ISM (dVSC), and will not consider any longer $\xi(r)$.

\section{EQUATIONS IN THE COMOVING FRAME}

Let us consider the universe in expansion with the characteristic scale factor $a(t)$. For the sake of simplicity, we modelise the galaxies by points of equal masses $m$, although they have a mass spectrum (it may be responsible for a multi-fractal structure, see Sylos Labini \& Pietronero 1996).

The present analysis can be generalised to galaxies of different masses following the lines of sec. IX [2]. We expect to come to this point in future work.

If the physical coordinates of the particles are $\vec{r}$, we can introduce the comoving coordinates $\vec{x}$ such that

$$
\vec{r}=a(t) \vec{x}
$$

The Lagrangian for a system of $N$ particles interacting only by their self-gravity can be written as

$$
L_{N}=\sum_{i=1}^{N}\left[\frac{m}{2} a(t)^{2} \dot{\vec{x}}_{i}^{2}-\frac{m}{a(t)} \phi\left(\vec{x}_{i}(t)\right)\right]
$$

where $\phi(\vec{x})$ is the gravitational potential in the comoving frame, determined by the Poisson equation

$$
\nabla^{2} \phi(\vec{x})=4 \pi G \rho(\vec{x}, t)
$$

and $\rho(\vec{x}, t)$ is the mass density. For our system of point particles,

$$
\rho(\vec{x}, t)=m \sum_{i=1}^{N} \delta\left(\vec{x}-\vec{x}_{i}(t)\right)
$$

and therefore the solution of the Poisson equation takes the form 


$$
\phi(\vec{x})=-G m \sum_{i=1}^{N} \frac{1}{\left|\vec{x}-\vec{x}_{i}(t)\right|} .
$$

The canonical momenta and Hamiltonian of the system are

$$
\begin{gathered}
\vec{p}_{i}=m a(t)^{2} \dot{\vec{x}}_{i} \\
H_{N}=\sum_{i=1}^{N}\left[\frac{1}{2 m a(t)^{2}} \vec{p}_{i}^{2}+\frac{m}{a(t)} \phi\left(\vec{x}_{i}(t)\right)\right] \\
=\frac{1}{2 m a(t)^{2}} \sum_{i=1}^{N}{\overrightarrow{p_{i}}}^{2}-\frac{G m^{2}}{a(t)} \sum_{1 \leq l<j \leq N} \frac{1}{\left|\vec{x}_{l}-\vec{x}_{j}\right|}
\end{gathered}
$$

We see that the $N$-particle Hamiltonian in cosmological spacetime eq.(7.5) can be obtained from the Minkowski Hamiltonian $[a(t)=1]$ by making the replacements

$$
m \rightarrow m a(t)^{2} \quad, \quad G \rightarrow G a(t)^{-5}
$$

As a first approximation, we shall assume in the following that the characteristic time of the particle motions under the gravitational self-interaction are shorter than the time variation of $a(t)$. We can then consider that this system of self-gravitating particles is at any time in approximate thermal equilibrium. This hypothesis is true of course for structures that have already decoupled from the expansion, and are truly self-gravitating and virialised. It could be also valid for the whole non-linear regime of the gravitational collapse. As for the linear regime, we know already that the primordial fluctuations are not forgotten in the large-scale structures, and therefore the resulting correlations will depend on initial conditions, and not be entirely determined by self-gravity.

The above assumption introduces a natural upper limit in the scales concerned by the theory developped below. Similarly to the case of the interstellar medium, the fractal structure considered is bounded by a short distance cut-off and by a large-scale limit as well (dVSC).

The short distance cut-off corresponds to the appearence of other physics at short scale, essentially dissipative, which we do not need to introduce. In addition, the short distance cut-off avoids the gravo-thermal catastrophe. For the ISM, the cut-off was naturally the size of the smaller fragments, of the order of the Jeans length. Here the cut-off corresponds also to the size of the 'particles' considered, i.e. the galaxy size, below which another physics steps in, related to stellar formation and radiation.

The fact that in the catalogs, we are observing in projection large-scale structures at different epochs, with different values of the scale factor $a(t)$, could slightly modify the fractal dimension. Even though fractal structures are self-similar, and scale-independent, the largest scales are systematically observed at a younger epoch where the contrast has not grown up as high as today. This evolution effect however should be significant only at high redshift $(>1)$, and the present catalogs are not yet statistically robust so far back in time (the average redshift of optical catalogs is about 0.1 ). 


\section{APPLICATION OF RENORMALIZATION GROUP THEORY}

As in all scale-independent problems, where the fluctuations cannot be represented by analytical functions, the renormalization group theory developped in the 1970's for the study of critical phenomena, appears here perfectly adapted (e.g. [15]). We can consider the fractal structure of the Universe as the critical state of a system, where fluctuations develop at any scale, with a very large correlation length (asymptotically infinite). The fluctuations that are distributed as a fractal of dimension $D$ are the large-scale structures of the universe (cf. |71|).

We generalise now the study of an $N$-body system only interacting through their own self-gravity to the case where the bodies are on a cosmological background.

Another approach has been proposed for galaxy correlations (not for ISM) [27], but it yields different critical exponents.

Let us apply the theory to the system of galaxy points, already defined in the previous section. Since they are considered in approximate thermal equilibrium, we will use the grand canonical ensemble, that also allows a variable number of particles. The grand partition function of the system can be written as in eq.3.1) where now $H_{N}$ is given by eq.(7.5).

The functional representation for the grand partition function can be easily generalized for an arbitrary scale factor $a(t)$. After the changes specified above in eq.(7.6), the local action becomes

$$
S[\phi(.)] \equiv \frac{a(t)}{T_{e f f}} \int d^{3} x\left[\frac{1}{2}(\nabla \phi)^{2}-\mu^{2} a(t)^{2} e^{\phi(\vec{x})}\right]
$$

Notice that all quantities depend on time through the scale factor $a(t)$ only. There is no integration over $t$.

The mass parameter $\mu$ in the $\phi$-theory gets effectively multiplied by the scale factor $a(t)$. Since the Jeans length $d_{J} \simeq \mu^{-1}$ according to eq.(2.14), in comoving coordinates $d_{J}$ effectively becomes

$$
d_{J}=\sqrt{\frac{12}{\pi}} \frac{1}{\mu a(t)}
$$

as one could have expected.

On the other hand, the dimensionless coupling constant

$$
g^{2}=\mu T_{e f f}
$$

is unchanged by the replacements of eq.(7.6).

Therefore, for any fixed time $t$ we find the same scaling behaviour, after making the replacement

$$
\mu \rightarrow \mu a(t)
$$

and keeping the coupling $g$ unchanged.

Thus, the renormalisation group and finite size scaling analysis of secs. IVC and IVD apply without essential changes to the galaxy distributions. Namely, the mass fluctuations $\Delta M(R)$ inside a volume $R$,

$$
(\Delta M(R))^{2} \equiv<M^{2}>-<M>^{2}
$$


will scale as

$$
\Delta M(R) \sim R^{\frac{1}{\nu}}
$$

The scaling exponent $\nu$ can then be identified as before with the inverse Haussdorf (fractal) dimension $D$ of the system

$$
D=\frac{1}{\nu}
$$

As usual in the theory of critical phenomena, there are only two independent critical exponents. All exponents can be expressed in terms of two of them: for instance the fractal dimension $D=1 / \nu$, and the independent exponent $\eta$, which usually governs the spinspin correlation functions. The exponent $\eta$ appears here in the $\phi$-field correlator (dVSC), describing the gravitational potential, that scales as

$$
<\phi(\vec{r})>\sim r^{-\frac{1}{2}(1+\eta)}
$$

The values of the critical exponents depend on the fixed point that governs the long range behaviour of the system.

The value of the dimensionless coupling constant $g^{2}=\mu T_{\text {eff }}$ should decide whether the fixed point chosen by the system is the mean field (weak coupling) or the Ising one (strong

coupling). At the tree level, we estimate $g \approx \frac{5}{\sqrt{N}}$, where $N$ is the number of points in a Jeans volume $d_{J}^{3}$. The coupling constant appears then of the order of 1 , and we cannot settle this question without effective computations of the renormalisation group equations. At this point, the predicted fractal dimension $D$ should be between 1.585 and 2 .

\section{A. Three point and higher correlations}

Our approach allows to compute higher order correlators without any extra assumption. The two and three point densities,

$$
\begin{gathered}
D\left(\vec{r}_{1}, \vec{r}_{2}\right) \equiv<n\left(\vec{r}_{1}\right) n\left(\vec{r}_{2}\right)> \\
D\left(\vec{r}_{1}, \vec{r}_{2}, \vec{r}_{3}\right) \equiv<n\left(\vec{r}_{1}\right) n\left(\vec{r}_{2}\right) n\left(\vec{r}_{3}\right)>
\end{gathered}
$$

can be expressed as follows in terms of the correlation functions:

$$
\begin{aligned}
D\left(\vec{r}_{1}, \vec{r}_{2}\right) & =n_{1} n_{2}+C_{12} \\
D\left(\vec{r}_{1}, \vec{r}_{2}, \vec{r}_{3}\right) & =n_{1} n_{2} n_{3}+n_{1} C_{23}+n_{3} C_{12}+n_{2} C_{13}+C_{123} .
\end{aligned}
$$

Here,

$$
n_{i} \equiv<n\left(\vec{r}_{i}\right)>\quad, i=1,2,3,
$$

and $C_{i j}$ and $C_{i j k}$ are the two and three point correlation functions, respectively,

$$
\begin{gathered}
C_{i j} \equiv C\left(\vec{r}_{i}, \vec{r}_{j}\right) \\
C_{i j k} \equiv C\left(\vec{r}_{i}, \vec{r}_{j}, \vec{r}_{k}\right)
\end{gathered}
$$


The behaviour of $n_{i}, C_{i j}$ and $C_{i j k}$ in the scaling regime follow from the renormalisation group equations at criticality (de Vega, Sánchez \& Combes, in preparation). If we do not impose homogeneity at all scales, we find,

$$
\begin{array}{r}
<n(\vec{r})>\simeq A r^{D-3}, \\
C\left(\vec{r}_{1}, \vec{r}_{2}\right) \stackrel{r_{1} \gg r_{2}}{\simeq} B r_{1}^{2(D-3)}, \\
C\left(\vec{r}_{1}, \vec{r}_{2}, \vec{r}_{3}\right) \stackrel{r_{1}>>r_{2}, r_{3}}{\simeq} C r_{1}^{3(D-3)},
\end{array}
$$

where $A, B$ and $C$ are constants and $D=1 / \nu$.

We can now derive the three point density behaviour when one point, say $\vec{r}_{1}$, is far away from the other two. We find from eqs.(8.4) and (8.5),

$$
\begin{aligned}
& D\left(\vec{r}_{1}, \vec{r}_{2}\right) \stackrel{r_{1} \gg r_{2}}{\simeq} A r_{1}^{D-3} n_{2}+B r_{1}^{2(D-3)}, \\
& D\left(\vec{r}_{1}, \vec{r}_{2}, \vec{r}_{3}\right) \stackrel{r_{1}>r_{2}, r_{3}}{\simeq} A r_{1}^{D-3}\left(n_{2} n_{3}+C_{23}\right) \\
&+B r_{1}^{2(D-3)}\left(n_{2}+n_{3}\right)+C r_{1}^{3(D-3)}
\end{aligned}
$$

Notice that this expression is dominated by the first term since $D-3<0$.

Higher point distributions can be treated analogously in our approach. We find that the dominant behaviour in the $N$-points density is

$$
C\left(\vec{r}_{1}, \vec{r}_{2}, \ldots, \vec{r}_{N}\right) \stackrel{r_{1}>>r_{i}, 2 \leq i \leq N}{\sim} r_{1}^{N(D-3)}
$$

Notice that when homogeneity is assumed to hold over all scales, the critical behaviour of the $N$-point correlation function involves a factor $r_{1}^{(N-1)(D-3)}$, 49.

Eqs.8.6-8.7) are qualitatively similar, although not identical, to the behaviour inferred assuming the factorized hierarchical Ansatz (fhA), (Balian \& Schaeffer 1989). That is,

$$
\begin{aligned}
D\left(\vec{r}_{1}, \vec{r}_{2}\right)^{f h A} & =\bar{n}^{2}\left(1+b r_{12}^{D-3}\right)^{r_{1} \gg>r_{2}} \bar{n}^{2}\left(1+r_{1}^{D-3}\right), \\
D\left(\vec{r}_{1}, \vec{r}_{2}, \vec{r}_{3}\right)^{f h A} & =\bar{n}^{3}\left\{1+b\left(r_{12}^{D-3}+r_{13}^{D-3}+r_{23}^{D-3}\right)\right. \\
& \left.+\quad Q_{3}\left[r_{12}^{D-3} r_{13}^{D-3}+r_{12}^{D-3} r_{23}^{D-3}+r_{13}^{D-3} r_{23}^{D-3}\right]\right\} \\
& \stackrel{r_{1} \gg>r_{2}, r_{3}}{{ }_{n}^{3}}\left[1+b r_{23}^{D-3}+2 r_{1}^{D-3}\left(b+Q_{3} r_{23}^{D-3}\right)+Q_{3} r_{1}^{2(D-3)}\right] .
\end{aligned}
$$

where $r_{12} \equiv\left|\vec{r}_{1}-\vec{r}_{2}\right|$ and so on. $\quad b$ and $Q_{3}$ are constants. Notice that in the factorized hierarchical Ansatz, the fractal dimension $D$ is not predicted but it is a free parameter.

We see that the dominant behaviours in eqs. (8.6) and (8.8) are similar in case the scaling exponents $D-3$ are the same. 


\section{DISCUSSION}

In previous sections we ignored gravitational forces external to the gas like stars etc. Adding a fixed external mass density $\rho_{\text {ext }}(\vec{r})$ amounts to introduce an external source

$$
J(\vec{r})=-T_{\text {eff }} \rho_{\text {ext }}(\vec{r})
$$

in eq.(3.13). Such term will obviously affect correlation functions, the mass density, etc. except when we look at the scaling behaviour which is governed by the critical point. That is, the values we find for the scaling exponents $d_{H}$ and $q$ are stable under external perturbations.

We considered all atoms with the same mass in the gravitational gas. It is easy to generalize the transformation into the $\phi$-field presented in section II for a mixture of several kinds of atoms. Let us consider $n$ species of atoms with masses $m_{a}, 1 \leq a \leq n$. Repeating the steps from eq.(3.1) to (3.11) yields again a field theory with a single scalar field but the action now takes the form

$$
S[\phi(.)] \equiv \frac{1}{T_{e f f}} \int d^{3} x\left[\frac{1}{2}(\nabla \phi)^{2}-\sum_{a=1}^{n} \mu_{a}^{2} e^{\frac{m_{a}}{m} \phi(\vec{x})}\right],
$$

where

$$
\mu_{a}^{2}=\sqrt{\frac{2}{\pi}} z_{a} G m_{a}^{3 / 2} m^{2} \sqrt{T},
$$

and $m$ is just a reference mass.

Correlation functions, mass densities and other observables will obviously depend on the number of species, their masses and fugacities but it is easy to see that the fixed points and scaling exponents are exactly the same as for the $\phi$-field theory (3.9)-(3.10).

We want to notice that there is an important difference between the behaviour of the gravitational gas and the spin models (and all other statistical models in the same universality class). For the gravitational gas we find scaling behaviour for a full range of temperatures and couplings. For spin models scaling only appears at the critical value of the temperature. At the critical temperature the correlation length $\xi$ is infinite and the theory is massless. For temperatures near the critical one, i. e. in the critical domain, $\xi$ is finite (although very large compared with the lattice spacing) and the correlation functions decrease as $\sim e^{-r / \xi}$ for large distances $r$. Fluctuations of the relevant operators support perturbations which can be interpreted as massive excitations. Such (massive) behaviour does not appear for the gravitational gas. The ISM correlators scale exhibiting power-law behaviour. This feature is connected with the scale invariant character of the Newtonian force and its infinite range.

The hypothesis of strict thermal equilibrium does not apply to the ISM as a whole where temperatures range from 5 to $50 \mathrm{~K}$ and even $1000 \mathrm{~K}$. However, since the scaling behaviour is independent of the temperature, it applies to each region of the ISM in thermal equilibrium. Therefore, our theory applies provided thermal equilibrium holds in regions or clouds. 
We have developped here the theory of a gravitationally interacting ensemble of bodies at a fixed temperature. In a real situation like the ISM, gravitational perturbations from external masses, as well as other perturbations are present. We have shown that the scaling solution is stable with respect to the gravitational perturbations. It is well known that solutions based on a fixed point are generally quite robust.

Our theory especially applies to the interstellar medium far from star forming regions, which can be locally far from thermal equilibrium, and where ionised gas at $10^{4} \mathrm{~K}$ together with coronal gas at $10^{6} \mathrm{~K}$ can coexist with the cold interstellar medium. In the outer parts of galaxies, devoid of star formation, the ideal isothermal conditions are met [7]. Inside the Galaxy, large regions satisfy also the near isothermal criterium, and these are precisely the regions where scaling laws are the best verified. Globally over the Galaxy, the fraction of the gas in the hot ionised phase represents a negligible mass, a few percents, although occupying a significant volume. Hence, this hot ionised gas is a perturbation which may not change the fixed point behaviour of the thermal self-gravitating gas.

In ref. [27] a connection between a gravitational gas of galaxies in an expanding universe and the Ising model is conjectured. However, the unproven identification made in ref. 27] of the mass density contrast with the Ising spin leads to scaling exponents different from ours.

Concerning the galaxy distributions, different scaling behaviours show up depending whether the system is homogeneous or not at large distances. The homogeneity property brings extra information which is not contained in the fundamental gravitational interaction. In condensed matter systems, such homogeneity hypothesis is easily verified by experiments. The homogeneity of the universe at large scales is a much more controversial issue [55,57].

Under the homogeneity hypothesis we find for the galaxy-galaxy correlator defined as in [55],

$$
\xi(r) \equiv \frac{<\rho\left(\vec{r}_{0}\right) \rho\left(\vec{r}_{0}+\vec{r}\right)>}{<\rho>^{2}}-1 \sim r^{-\gamma}
$$

where $\gamma=6-2 d_{H}$. This gives $\gamma_{\text {mean field }}=2$ and $\gamma_{\text {Ising }}=2.830 \ldots$ Such numbers should be compared with the customary value $\gamma=1.8$ obtained from the observations [55].

In the inhomogeneous regime we find for the galaxy-galaxy correlations considered in ref. 57,

$$
D(r)=<\rho\left(\vec{r}_{0}\right) \rho\left(\vec{r}_{0}+\vec{r}\right)>\sim r^{-\Gamma}
$$

where $\Gamma=3-d_{H}$. This gives $\Gamma_{\text {mean field }}=1$ and $\Gamma_{\text {Ising }}=1.415 \ldots$. Such numbers should be compared with the observed value for $\Gamma \simeq 1$ [57] (obtained for $r>30 h^{-1} \mathrm{Mpc}$ ).

In conclusion, our renormalization group results seem to fit better with the analysis of the observations made in ref. [57] than with the standard lore [55]. However, our predictions do not disagree in a dramatic way with the standard analysis of the observations. In both cases, mean field exponents give the better agreement with observations.

Nuevas conclusiones:

The statistical analysis of the most recent galaxy catalogs, without the assumption of homogeneity at a scale smaller than the catalog depth, has determined that the universe has a fractal structure at least up to $\approx 100 h^{-1} \mathrm{Mpc}$ (Sylos Labini et al 1996). The analysis 
in terms of conditional density has revealed that the fractal dimension is between $D=1.5$ and 2 (Di Nella et al 1996, Sylos Labini \& Amendola 1996). We apply a theory that we have developped to explain the fractal structure of the interstellar medium (dVSC), which has the same dimension $D$. The physics is based on the self-gravitating interaction of an ensemble of particles, over scales limited both at short and large distances. The shortdistance cut-off is brought by other physical processes including dissipation. The long-range limit is fixed by the expansion time-scale. In-between, the system is assumed in approximate thermal equilibrium. The dynamical range of scales involved in this thermodynamic quasiequilibrium is at present limited to 3-4 orders of magnitude, but will increase with time.

The critical exponents found in the theory do not depend on the conditions at the cutoff, which determine only the amplitudes. The theory is based on the statistical study of the gravitational field: it is shown that the partition function of the N-body ensemble is equivalent to the partition function of a single scalar field, with a local action. This allows to use field theory methods and the renormalisation group to find the scaling behaviour. We find scaling behaviour for a full range of temperatures and couplings. The theory then predicts for the system a fractal dimension $D=1.585$ for the Ising fixed point, or $D=2$ in the case of the mean-field fixed point. Both are compatible with the available observations. The $N$-points density correlators are predicted to scale with exponent $(N-1)(D-3)$ when $r_{1}>>r_{i}, 2 \leq i \leq N$. That is, $-(N-1)$ for the mean field, or $-1.415(N-1)$ for the Ising point.

We predict in addition a critical exponent $-\frac{1}{2}(1+\eta)$ for the gravitational potential: that is, -0.500 for mean field or -0.519 for the Ising fixed point. 


\section{REFERENCES}

[1] H. J. de Vega, N. Sánchez and F. Combes, Nature, 383, 56 (1996).

[2] H. J. de Vega, N. Sánchez and F. Combes, Phys. Rev. D54, 6008 (1996) (dVSC).

[3] H. J. de Vega, N. Sánchez and F. Combes, 'The fractal structure of the universe: a new field theory approach', preprint.

[4] R. B. Larson, M.N.R.A.S. 194, 809 (1981)

[5] J. M. Scalo, in 'Interstellar Processes', D.J. Hollenbach and H.A. Thronson Eds., D. Reidel Pub. Co, p. 349 (1987).

[6] R. B. Larson, M.N.R.A.S. 256, 641 (1992)

[7] D. Pfenniger, F. Combes, L. Martinet, A\&A 285, 79 (1994)

D. Pfenniger, F. Combes, A\&A 285, 94 (1994)

[8] L. Landau and E. Lifchitz, Mécanique des Fluides, Eds. MIR, Moscou 1971.

[9] C.F. von Weizsäcker, ApJ, 114, 165 (1951).

[10] L. P. Kadanoff, 'From Order to Chaos', World. Sc. Pub.(1993).

[11] S. Edward and A. Lenard, J. M. P. 3, 778 (1962).

S. Albeverio and R. Høegh-Krohn, C. M. P. 30, 171 (1973).

[12] R. L. Stratonovich, Doklady, 2, 146 (1958).

J. Hubbard, Phys. Rev. Lett, 3, 77 (1959).

J. Zittartz, Z. Phys., 180, 219 (1964).

[13] L. D. Landau and E. M. Lifchitz, Physique Statistique, 4ème édition, Mir-Ellipses, 1996.

[14] S. Samuel, Phys. Rev. D 18, 1916 (1978).

[15] Wilson K.G., Kogut, J., Phys. Rep. 12, 75 (1974).

K. G. Wilson, Rev. Mod. Phys. 47, 773 (1975) and Rev. Mod. Phys. 55, 583 (1983).

[16] Phase transitions and Critical Phenomena vol. 6,

C. Domb \& M. S. Green, Academic Press, 1976.

[17] J. J. Binney, N. J. Dowrick, A. J. Fisher and M. E. J. Newman,

The Theory of Critical Phenomena, Oxford Science Publication, 1992.

[18] See for example,

H. Stanley in Fractals and Disordered Systems,

A. Bunde and S. Havlin editors, Springer Verlag, 1991.

[19] See for example, W. C. Saslaw, 'Gravitational Physics of stellar and galactic systems', Cambridge Univ. Press, 1987.

[20] A. Hasenfratz and P. Hasenfratz, Nucl.Phys. B270, 687 (1986).

T. R. Morris, Phys. Lett. B329, 241 (1994) and B334, 355 (1994).

[21] S. Chandrasekhar, 'An Introduction to the Study of Stellar Structure', Chicago Univ. Press, 1939.

[22] G. Horwitz and J. Katz, Ap. J. 222, 941 (1978) and 223, 311 (1978).

J. Katz, G. Horwitz and A. Dekel, Ap. J. 223, 299 (1978).

[23] H. J. de Vega, N. Sánchez, B. Semelin and F. Combes, in preparation.

[24] S.C. Kleiner, R.L. Dickman,

ApJ 286, 255 (1984), ApJ 295, 466 (1985), ApJ 312, 837 (1987)

[25] A. M. Polyakov, Phys. Lett. B103, 207 (1981).

[26] J. Ambjørn and Y. Watabiki, Nucl. Phys. B 445, 129 (1995).

J. Ambjørn, J. Jurkiewicz and Y. Watabiki, Nucl. Phys. B 454, 313 (1995).

Y. Watabiki, hep-th/9605185. 
[27] J. Pérez Mercader, T. Goldman, D. Hochberg and R. Laflamme, astro-ph/9506127 and LAEFF-96/06.

Hochberg D., Pérez Mercader J. , Gen. Relativity and Gravit. 28, 1427 (1996).

[28] Abell G.O.: 1958, ApJS 3, 211

[29] Balian R., Schaeffer R.: 1988, ApJ 335, L43

[30] Balian R., Schaeffer R.: 1989, A\&A 226, 373

[31] Bouchet F.R., Schaeffer R., Davis M.: 1991, ApJ 383, 19

[32] Castagnoli C., Provenzale A.: 1991, A\&A 246, 634

[33] Coleman P.H., Pietronero L., Sanders R.H.: 1988, A\&A 200, L32

[34] Coleman P.H., Pietronero L.: 1992, Phys. Rep. 231, 311

[35] Colombi S., Bouchet F.R., Hernquist L.: 1996, ApJ 465, 14

[36] Davis M.A., Peebles P.J.E.: 1977, ApJS 34, 425

[37] Davis M.A., Peebles P.J.E.: 1983, ApJ 267, 465

[38] Davis M.A., Meiksin M.A., Strauss L.N., da Costa and Yahil A.: 1988, ApJ 333, L9

[39] de Lapparent V., Geller M.J., Huchra J.P.: 1986, ApJ 302, L1

[40] de Vaucouleurs G.: 1960, ApJ 131, 585

[41] de Vaucouleurs G.: 1970, Science 167, 1203

[42] Di Nella H., Montuori M., Paturel G., Pietronero L., Sylos Labini F.: 1996, A\&A 308, L33

[43] Dubrulle B., Lachieze-Rey M.: 1994, A\&A 289, 667

[44] Einasto J.: 1989, in 'Astronomy, cosmology and fundamental physics', Proc. of the 3rd ESO-CERN Symposium, Dordrecht, Kluwer, p. 231

[45] Falgarone, E., Phillips, T.G., Walker, C.K.: 1991, ApJ 378, 186

[46] Geller M.J., Huchra J.P.: 1989, Science 246, 897

[47] Hamilton A.J.S.: 1993, ApJ 417, 19

[48] Itoh M., Inagaki S., Saslaw W.C.: 1993, ApJ 403, 459

[49] Itzykson C. , Drouffe, J.-M., 'Théorie Statistique des Champs', Inter/CNRS, 1989, Paris.

[50] Lin H. et al: 1996, ApJ 471, 617

[51] Mandelbrot B.B.: 1975, 'Les objets fractals', Paris, Flammarion

[52] Mandelbrot B.B.: 1982, 'The fractal geometry of nature', New York: Freeman

[53] Martinez V.J., Paredes S., Saar E.: 1993, MNRAS 260, 365

[54] Ostriker J.P.: 1993, ARAA 31, 689

[55] Peebles P.J.E.: 1980, 'The Large-scale structure of the Universe', Princeton Univ. Press Peebles P.J.E.: 1993, 'Principles of physical cosmology' Princeton Univ. Press

[56] Pfenniger D., Combes F.: 1994, A\&A 285, 94

[57] Pietronero L.: 1987, Physica A, 144, 257

Pietronero L., Montuori M., Sylos Labini F.: 1997, in 'Critical Dialogs in Cosmology', ed. N. Turok, astro-ph/9611197

[58] Postman M., Geller M.J., Huchra J.P.: 1986, AJ 91, 1267

[59] Postman M., Huchra J.P., Geller M.J.: 1992, ApJ 384, 404

[60] Saslaw W.C., Hamilton A.J.S.: 1984, ApJ 276, 13

[61] Saslaw W.C., Fang F.: 1996, ApJ 460, 16

[62] Scott E.L., Shane S.D., Swanson M.D.: 1954, ApJ 119, 91

[63] Shapley H.: 1934, MNRAS 94, 791

[64] Sheth R.K., Saslaw W.C.: 1996, Apj 470, 78 
[65] Smoot G., et al: 1992, ApJ 396, L1

[66] Soneira R.M., Peebles P.J.E.: 1978, AJ 83, 845

[67] Sylos Labini F.: 1994, ApJ 433, 464

[68] Sylos Labini F., Amendola L.: 1996, ApJ 438, L1

[69] Sylos Labini F., Pietronero L.: 1996, ApJ 469, 26

[70] Sylos Labini F., Gabrielli A., Montuori M., Pietronero L.: 1996, Physica A 226, 195

[71] Totsuji H., Kihara T., PASJ 21, 221 (1969).

[72] White S.D.M.: 1979, MNRAS 186, 145 